\title{
The Cauchy Problem for a Dissipative Periodic 2-Component Degasperis-Procesi System
}

\author{
Sen Ming, ${ }^{1}$ Han Yang, ${ }^{1}$ and Ls Yong ${ }^{2}$ \\ ${ }^{1}$ School of Mathematics, Southwest Jiaotong University, Chengdu 610031, China \\ ${ }^{2}$ Department of Mathematics, Southwestern University of Finance and Economics, Chengdu 611130, China
}

Correspondence should be addressed to Han Yang; hanyang95@263.net

Received 28 March 2014; Accepted 16 June 2014; Published 23 July 2014

Academic Editor: Sazzad Hossien Chowdhury

Copyright (c) 2014 Sen Ming et al. This is an open access article distributed under the Creative Commons Attribution License, which permits unrestricted use, distribution, and reproduction in any medium, provided the original work is properly cited.

The dissipative periodic 2-component Degasperis-Procesi system is investigated. A local well-posedness for the system in Besov space is established by using the Littlewood-Paley theory and a priori estimates for the solutions of transport equation. The wavebreaking criterions for strong solutions to the system with certain initial data are derived.

\section{Introduction}

We consider the following dissipative periodic 2-component Degasperis-Procesi system:

$$
\begin{aligned}
& u_{t}-u_{x x t}+4 u u_{x}+\lambda_{1}\left(u-u_{x x}\right)+c \rho \rho_{x}=3 u_{x} u_{x x}+u u_{x x x}, \\
& t>0, \quad x \in \mathbb{S}, \\
& \rho_{t}+u \rho_{x}+2 u_{x} \rho+\lambda \rho=0, \quad t>0, x \in \mathbb{S}, \\
& u(t, x)=u(t, x+1), \quad \rho(t, x)=\rho(t, x+1), \\
& t \geq 0, \quad x \in \mathbb{S}, \\
& u(0, x)=u_{0}(x), \quad \rho(0, x)=\rho_{0}(x), \quad x \in \mathbb{S},
\end{aligned}
$$

where $\lambda$ and $\lambda_{1}$ are nonnegative constants, $c \in \mathbb{R},\left(u_{0}, \rho_{0}\right) \in$ $B_{p, r}^{s}(\mathbb{S}) \times B_{p, r}^{s-1}(\mathbb{S})$ with $s>\max (3 / 2,1+1 / p)$, and $\mathbb{S}=\mathbb{R} / \mathbb{Z}$ denotes the unit circle.

In system (1), if $\lambda_{1}=\rho=0$, we get the classical Degasperis-Procesi equation [1]

$$
u_{t}-u_{x x t}+4 u u_{x}=3 u_{x} u_{x x}+u u_{x x x}
$$

where $u(t, x)$ represents the fluid velocity at time $t$ in $x$ direction (or equivalently the height of water's free surface above a flat bottom). The nonlinear convection term $u u_{x}$ causes the steepening of the wave form. The nonlinear dispersion effect term $3 u_{x} u_{x x}+u u_{x x x}$ makes the wave form spread.

Equation (2) has attracted many researchers to discover its dynamics properties [2-15]. For example, Degasperis et al. [2] proved the formal integrability by constructing a Lax pair. They showed that (2) has bi-Hamiltonian structure with an infinite sequence of conserved quantities and admits exact peakon solutions which are analogous to the Camassa-Holm peakons. The asymptotic accuracy of (2) is the same as that of Camassa-Holm equation. Dullin et al. [3] showed that the Degasperis-Procesi equation can be derived from the shallow water elevation equation by an appropriate Kodama transformation. Lin and Liu [16] proved the stability of peakons for (2) under certain assumptions. In [17], Yin proved the local well-posedness for (2) with initial data $u_{0} \in$ $H^{s}(\mathbb{R})(s>3 / 2)$ and also derived the precise blow-up scenarios for the solutions. The global existence of strong solutions and global weak solutions to (2) are studied in [18]. Escher and Kolev [4] and Escher and Seiler [5] showed that the Degasperis-Procesi equation can be reformulated as a nonmetric Euler equation on the diffeomorphism group of the circle. Vakhnenko and Parkes [7] derived periodic and solitary wave solutions to (2). Lundmark and Szmigielski [8] investigated multipeakon solutions to (2). The shock wave solutions to (2) were obtained in [9]. Although the Degasperis-Procesi equation is similar to the Camassa-Holm 
equation in many aspects, especially in the structure of equation, there are some differences between the two equations. One of the famous features of Degasperis-Procesi equation is that it not only has peakon solutions $u(t, x)=c e^{-|x-c t|}$ with $c>0$ [2] and periodic peakon solutions [18] but also has shock peakons [9] and periodic shock waves [19].

In general, it is difficult to avoid the energy dissipation mechanisms in a real world. Thus different types of solutions for the dissipative Degasperis-Procesi equation have been investigated. For example, Guo et al. [20] studied the dissipative Degasperis-Procesi equation

$$
u_{t}-u_{x x t}+4 u u_{x}+\lambda\left(u-u_{x x}\right)=3 u_{x} u_{x x}+u u_{x x x},
$$

where $\lambda\left(u-u_{x x}\right)(\lambda>0)$ is the dissipative term. They obtained the global existence of weak solutions. Wu and Yin [21] established blow-up solutions and analyzed the decay of solutions to (3). In [22], the authors studied the long time behavior of solutions to (3). Guo [23] established the local well-posedness, blow-up scenario, global existence of solutions, and persistence properties for strong solutions to (3).

On the other hand, many researchers have studied the integrable multicomponent generalizations of the Degasperis-Procesi equation [24-29]. For example, Yan and Yin [28] investigated the 2-component Degasperis-Procesi system

$$
\begin{gathered}
u_{t}-u_{x x t}+4 u u_{x}+c \rho \rho_{x}=3 u_{x} u_{x x}+u u_{x x x}, \\
t>0, \quad x \in \mathbb{R}, \\
\rho_{t}+u \rho_{x}+2 u_{x} \rho=0, \quad t>0, \quad x \in \mathbb{R}, \\
u(0, x)=u_{0}(x), \quad x \in \mathbb{R}, \\
\rho(0, x)=\rho_{0}(x), \quad x \in \mathbb{R},
\end{gathered}
$$

where $c \in \mathbb{R}$. They established the local well-posedness for system (4) in Besov space $B_{p, r}^{s}(\mathbb{R}) \times B_{p, r}^{s-1}(\mathbb{R})$ with $s>\max (1+$ $1 / p, 3 / 2)$ and also derived the precise blow-up scenarios for strong solutions in Sobolev space $H^{s}(\mathbb{R}) \times H^{s-1}(\mathbb{R})$ with $s>3 / 2$. Zhou et al. [27] investigated the traveling wave solutions of the 2-component Degasperis-Procesi system. Jin and Guo [25] established the local well-posedness, blow-up criterions and the persistence properties of strong solutions to the system in $H^{s}(\mathbb{R}) \times H^{s}(\mathbb{R})$ with $s>5 / 2$.

Recently, a large amount of literature was devoted to the 2 component Camassa-Holm system [30-39]. For example, Hu [40] studied the dissipative periodic 2-component CamassaHolm system

$$
\begin{array}{r}
u_{t}-u_{x x t}+3 u u_{x}+\lambda\left(u-u_{x x}\right)+\rho \rho_{x}=2 u_{x} u_{x x}+u u_{x x x} \\
t>0, \quad x \in \mathbb{S}
\end{array}
$$

$$
\begin{array}{r}
\rho_{t}+u \rho_{x}+u_{x} \rho+\lambda \rho=0, \quad t>0, \quad x \in \mathbb{S}, \\
u(t, x)=u(t, x+1), \quad \rho(t, x)=\rho(t, x+1), \\
t \geq 0, \quad x \in \mathbb{S}, \\
u(0, x)=u_{0}(x), \quad \rho(0, x)=\rho_{0}(x), \quad x \in \mathbb{S},
\end{array}
$$

where $\lambda>0$. The author not only established the local wellposedness for system (5) in Besov space $B_{p, r}^{s}(\mathbb{S}) \times B_{p, r}^{s-1}(\mathbb{S})$ with $s>\max (1+1 / p, 3 / 2)$ but also presented global existence of solutions and the exact blow-up scenarios of strong solutions in Sobolev space $H^{s}(\mathbb{S}) \times H^{s-1}(\mathbb{S})$ with $s>$ $3 / 2$. It was shown in [41] that the dissipative Camassa-Holm, Degasperis-Procesi, Hunter-Saxton, and Novikov equations can be reduced to their nondissipative versions by means of an exponentially time dependent scaling.

Motivated by the work in $[20,28,32,40-43]$, we study the dissipative periodic 2-component Degasperis-Procesi system (1). We note that the Cauchy problem of system (1) in Besov space has not been discussed yet. One of the difficulties is that we can not obtain the estimates for $\int_{\mathbb{R}}\left(u^{2}+u_{x}^{2}+\rho^{2}\right) d x$, which is a conserved quantity playing a key role in studying the blow-up phenomenon of the 2-component Camassa-Holm system $[32,33]$. However, this difficulty has been dealt with by establishing the estimates for $\|u(t)\|_{L^{\infty}}$, where $u$ is the first component of solution $(u, \rho)$ to system $(1)$. We state our main task with two aspects. Firstly, we establish the local wellposedness for system (1) in Besov space. Secondly, we present the precise blow-up criterions for strong solutions.

We rewrite system (1) as

$$
\begin{gathered}
u_{t}+u u_{x}=P(D)\left(\frac{3}{2} u^{2}+\frac{c}{2} \rho^{2}\right)-\lambda_{1} u, \\
t>0, \quad x \in \mathbb{S}, \\
\rho_{t}+u \rho_{x}=-2 u_{x} \rho-\lambda \rho, \quad t>0, \quad x \in \mathbb{S}, \\
u(t, x)=u(t, x+1), \quad \rho(t, x)=\rho(t, x+1), \\
t \geq 0, \quad x \in \mathbb{S}, \\
u(0, x)=u_{0}(x), \quad \rho(0, x)=\rho_{0}(x), \quad x \in \mathbb{S},
\end{gathered}
$$

where the operator $P(D)=-\partial_{x}\left(1-\partial_{x}^{2}\right)^{-1}$. We write the space

$$
\begin{aligned}
E_{p, r}^{\mathcal{s}}(T) & \left\{\begin{array}{c}
C\left([0, T] ; B_{p, r}^{s}(\mathbb{S})\right) \cap C^{1}\left([0, T] ; B_{p, r}^{s-1}(\mathbb{S})\right), \\
1 \leq r<\infty, \\
L^{\infty}\left([0, T] ; B_{p, \infty}^{s}(\mathbb{S})\right) \cap \operatorname{Lip}\left([0, T] ; B_{p, \infty}^{s-1}(\mathbb{S})\right), \\
r=\infty,
\end{array}\right.
\end{aligned}
$$

with $T>0, s \in \mathbb{R}, p \in[1, \infty], r \in[1, \infty]$.

The main results of this paper are presented as follows. 
Theorem 1. Let $1 \leq p, r \leq \infty, s>\max (3 / 2,1+1 / p)$, and $\left(u_{0}, \rho_{0}\right) \in B_{p, r}^{s}(\mathbb{S}) \times B_{p, r}^{s-1}(\mathbb{S})$. Then there exists a time $T>0$ such that the Cauchy problem (1) has a unique solution $(u, \rho) \epsilon$ $E_{p, r}^{s}(T) \times E_{p, r}^{s-1}(T)$. The map $\left(u_{0}, \rho_{0}\right) \rightarrow(u, \rho)$ is continuous from a neighborhood of $\left(u_{0}, \rho_{0}\right)$ in $B_{p, r}^{s}(\mathbb{S}) \times B_{p, r}^{s-1}(\mathbb{S})$ into $C\left([0, T] ; B_{p, r}^{s^{\prime}}(\mathbb{S})\right) \cap C^{1}\left([0, T] ; B_{p, r}^{s^{\prime}-1}(\mathbb{S})\right) \times C\left([0, T] ; B_{p, r}^{s^{\prime}-1}(\mathbb{S})\right) \cap$ $C^{1}\left([0, T] ; B_{p, r}^{s^{\prime}-2}(\mathbb{S})\right)$ for every $s^{\prime}<s$ when $r=\infty$ and $s^{\prime}=s$ whereas $r<\infty$.

Theorem 2. Let $\left(u_{0}, \rho_{0}\right) \in H^{s}(\mathbb{S}) \times H^{s-1}(\mathbb{S})$ with $s>3 / 2$ and $T<\infty$ is the maximal existence time of corresponding solution $(u, \rho)$ to system $(1)$. Then

$$
\int_{0}^{T}\left\|\partial_{x} u(\tau)\right\|_{L^{\infty}} d \tau=\infty
$$

Theorem 3. Let $\left(u_{0}, \rho_{0}\right) \in H^{s}(\mathbb{S}) \times H^{s-1}(\mathbb{S})$ with $s>3 / 2$ and $T<\infty$ is the maximal existence time of corresponding solution $(u, \rho)$ to system $(1)$. Then the solution $(u, \rho)$ blows up in finite time if and only if

$$
\lim \inf _{t \rightarrow T^{-}} \inf _{x \in \mathbb{S}} u_{x}(t, x)=-\infty .
$$

Theorem 4. Let $c \geq 0$ in system (1) and $\left(u_{0}, \rho_{0}\right) \in H^{s}(\mathbb{S}) \times$ $H^{s-1}(\mathbb{S})$ with $s>5 / 2$. Assume that $u_{0}$ is odd, $\rho_{0}$ is even, $u_{0 x}(0)<-\lambda_{1}$, and $\rho_{0}(0)=0$. Then the corresponding solution $(u, \rho)$ to system (1) blows up in finite time. More precisely, there exists $T \in\left(0,-1 /(1-\delta)\left(u_{0 x}(0)+\lambda_{1} / 2\right)\right]$ such that

$$
\lim \inf _{t \rightarrow T^{-}} u_{x}(t, 0)=-\infty .
$$

In addition, if $\rho_{0 x}\left(x_{0}\right) \neq 0$ with some $x_{0} \in \mathbb{S}$ satisfying $u_{0 x}\left(x_{0}\right)=\inf _{x \in \mathbb{S}} u_{0 x}(x)$, then there exists $T_{1} \in(0,-1 /(1-$ $\left.\delta)\left(u_{0 x}(0)+\lambda_{1} / 2\right)\right]$ such that

(i) $\lim \sup _{t \rightarrow T_{1}^{-}} \sup _{x \in \mathbb{S}} \rho_{x}(t, x)=+\infty$ if $\rho_{0 x}\left(x_{0}\right)>0$;

(ii) $\liminf \operatorname{int}_{t \rightarrow T_{1}^{-}} \inf _{x \in \mathbb{S}} \rho_{x}(t, x)=-\infty$ if $\rho_{0 x}\left(x_{0}\right)<0$,

where $\delta \in(0,1)$ such that $-\sqrt{\delta}\left[u_{0 x}(0)+\lambda_{1} / 2\right]=\lambda_{1} / 2$.

Theorem 5. Let $c \geq 0$ in system (1) and $\left(u_{0}, \rho_{0}\right) \in H^{s}(\mathbb{S}) \times$ $H^{s-1}(\mathbb{S})$ with $s>5 / 2$. Assume that $u_{0}$ and $\rho_{0}$ are odd, $u_{0 x}(0)<$ $-\lambda_{1}$. Then the corresponding solution $(u, \rho)$ to system (1) blows up in finite time. More precisely, there exists $T_{2} \in(0,-1 /(1-$ $\left.\delta)\left(u_{0 x}(0)+\lambda_{1} / 2\right)\right]$ such that

$$
\lim \inf _{t \rightarrow T_{2}^{-}} u_{x}(t, 0)=-\infty
$$

In addition, the inequalities hold:
(i) $\rho_{x}(t, 0)>\rho_{0 x}(0) e^{-\left(3 u_{0 x}(0)+\lambda\right) t}$ if $\rho_{0 x}(0)>0$;
(ii) $\rho_{x}(t, 0)<\rho_{0 x}(0) e^{-\left(3 u_{0 x}(0)+\lambda\right) t}$ if $\rho_{0 x}(0)<0$.

The remainder of this paper is organized as follows. In Section 2, several properties of Besov space and a priori estimates for solutions of transport equation are reviewed.
Section 3 is devoted to the proof of Theorem 1 . The proofs of Theorems 2, 3, 4, and 5 are presented in Section 4.

Notation. We denote the norm in Lebesgue space $L^{p}, 1 \leq p \leq$ $\infty$, by $\|\cdot\|_{L^{p}}$, the norm in Sobolev space $H^{s}, s \in \mathbb{R}$, by $\|\cdot\|_{H^{s}}$, and the norm in Besov space $B_{p, r}^{s}, s \in \mathbb{R}$, by $\|\cdot\|_{B_{p, r}^{s}}$. Since functions in all the spaces are over $\mathbb{S}$, for simplicity, we drop $\mathbb{S}$ in our notations if there is no ambiguity. We denote $a+=a+\varepsilon$, where $\varepsilon>0$ is a sufficiently small number.

\section{Preliminary}

This section is concerned with some basic facts in periodic Besov space and the theory of transport equation. One may check [33, 44-49] for more details.

Proposition 6 (see $[44,46]$ ). There exists a couple of smooth functions $(\chi, \varphi)$ valued in $[0,1]$, such that $\chi$ is supported in the ball $B=\{\xi \in \mathbb{R}|| \xi \mid \leq 4 / 3\}$, and $\varphi$ is supported in the ring $C=\{\xi \in \mathbb{R}|3 / 4 \leq| \xi \mid \leq 8 / 3\}$. Moreover,

$$
\begin{gathered}
\chi(\xi)+\sum_{q \in \mathbb{N}} \varphi\left(2^{-q} \xi\right)=1, \quad \forall \xi \in \mathbb{R}, \\
\operatorname{supp} \varphi\left(2^{-q} \cdot\right) \cap \operatorname{supp} \varphi\left(2^{-q^{\prime}} \cdot\right)=\emptyset, \quad \text { if }\left|q-q^{\prime}\right| \geq 2, \\
\operatorname{supp} \chi(\cdot) \cap \operatorname{supp} \varphi\left(2^{-q} \cdot\right)=\emptyset, \quad \text { if } q \geq 1 .
\end{gathered}
$$

Then, for all $u \in S^{\prime}(\mathbb{S})$, we define the nonhomogeneous dyadic blocks as follows:

$$
\begin{gathered}
\Delta_{q} u=0, \quad \text { if } q \leq-2 ; \\
\Delta_{-1} u=\sum_{\xi \in \mathbb{Z}} \chi(\xi) \widehat{u}(\xi) e^{2 \pi i x \xi} ; \\
\Delta_{q} u=\sum_{\xi \in \mathbb{Z}} \varphi\left(2^{-q} \xi\right) \widehat{u}(\xi) e^{2 \pi i x \xi}, \quad \text { if } q \geq 0 .
\end{gathered}
$$

Thus $u=\sum_{q \geq-1} \Delta_{q} u$, which is called the nonhomogeneous Littlewood-Paley decomposition of $u$.

Proposition 7 (see $[44,46]$ ). Let $s \in \mathbb{R}, 1 \leq p, r \leq \infty$. The nonhomogeneous periodic Besov space $B_{p, r}^{s}(\mathbb{S})$ is defined by $B_{p, r}^{s}(\mathbb{S})=\left\{f \in S^{\prime}(\mathbb{S}) \mid\|f\|_{B_{p, r}^{s}(\mathbb{S})}<+\infty\right\}$, where

$$
\|f\|_{B_{p, r}^{s}(\mathbb{S})}= \begin{cases}\left(\sum_{j=-1}^{\infty} 2^{j r s}\left\|\Delta_{j} f\right\|_{L^{p}}^{r}\right)^{1 / r}, & r<\infty, \\ \sup _{j \geq-1}^{j s}\left\|\Delta_{j} f\right\|_{L^{p}}, & r=\infty .\end{cases}
$$

Moreover, the low frequency cut-off $S_{q}$ is defined as $S_{q} u=$ $\sum_{p=-1}^{q-1} \Delta_{p} u$ for all $q \in \mathbb{N}$.

Proposition 8 (see $[44,49]$ ). Let $s \in \mathbb{R}, 1 \leq p, r, p_{1}, p_{2}, r_{1}, r_{2}$ $\leq \infty$; then consider the following.

(1) Density: $C_{c}^{\infty}$ is dense in $B_{p, r}^{s} \Leftrightarrow 1 \leq p, r<\infty$. 
(2) Embedding: $B_{p_{1}, r_{1}}^{s} \hookrightarrow B_{p_{2}, r_{2}}^{s-n\left(1 / p_{1}-1 / p_{2}\right)}$, if $p_{1} \leq p_{2}, r_{1} \leq$ $r_{2} . B_{p, r_{2}}^{s_{2}} \hookrightarrow B_{p, r_{1}}^{s_{1}}$ is locally compact if $s_{1} \leq s_{2}$.

(3) Algebraic properties: for all $s>0, B_{p, r}^{s} \cap L^{\infty}$ is an algebra. $B_{p, r}^{s}$ is an algebra $\Leftrightarrow B_{p, r}^{s} \hookrightarrow L^{\infty} \Leftrightarrow s>$ $n / p$ or $s \geq n / p$ and $r=1$.

(4) Complex interpolation: consider

$$
\begin{gathered}
\|f\|_{B_{p, r}^{\theta s_{1}+(1-\theta) s_{2}}} \leq C\|f\|_{B_{p, r}^{s_{1}}}^{\theta}\|f\|_{B_{p, r}^{s_{2}}}^{1-\theta}, \\
\forall f \in B_{p, r}^{s_{1}} \cap B_{p, r}^{s_{2}}, \quad \theta \in[0,1] .
\end{gathered}
$$

(5) Fatou's Lemma: if $\left(u_{n}\right)_{n \in \mathbb{N}}$ is bounded in $B_{p, r}^{s}$ and $u_{n} \rightarrow$ $u$ in $S^{\prime}(\mathbb{S})$, then $u \in B_{p, r}^{s}$ and

$$
\|u\|_{B_{p, r}^{s}} \leq \lim _{n \rightarrow \infty} \inf \left\|u_{n}\right\|_{B_{p, r}^{s}}
$$

(6) 1-D Morse-type estimates.

(i) For $s>0$,

$$
\|f g\|_{B_{p, r}^{s}} \leq C\left(\|f\|_{B_{p, r}^{s}}\|g\|_{L^{\infty}}+\|f\|_{L^{\infty}}\|g\|_{B_{p, r}^{s}}\right)
$$

(ii) For $s_{1} \leq 1 / p, s_{2}>(1 / p)\left(s_{2} \geq 1 / p\right.$ if $\left.r=1\right)$, and $s_{1}+s_{2}>0$, then

$$
\|f g\|_{B_{p, r}^{s_{1}}} \leq C\|f\|_{B_{p, r}^{s_{1}}}\|g\|_{B_{p, r}^{s_{2}}}
$$

(iii) In Sobolev space $H^{s}=B_{2,2}^{s}$, for $s>0$, we have

$$
\left\|f \partial_{x} g\right\|_{H^{s}} \leq C\left(\|f\|_{H^{s+1}}\|g\|_{L^{\infty}}+\|f\|_{L^{\infty}}\left\|\partial_{x} g\right\|_{H^{s}}\right) .
$$

(7) The lifting property: let $u \in S^{\prime}(\mathbb{S})$ and $\alpha \in \mathbb{R}$; then $u \in B_{p, r}^{s}$ if and only if

$$
\sum_{\xi \in \mathbb{Z}, \xi \neq 0}(i \xi)^{\alpha} \widehat{u}(\xi) e^{2 \pi i x \xi} \in B_{p, r}^{s-\alpha} .
$$

Lemma 9 (see [46]). Let $1 \leq p, r \leq \infty, s>-\min (1 / p, 1-$ $1 / p)$. Assume $f_{0} \in B_{p, r}^{s}, F \in L^{1}\left([0, T] ; B_{p, r}^{s}\right)$, and $\partial_{x} v \in$ $L^{1}\left([0, T] ; B_{p, r}^{s-1}\right)$ if $s>1+1 / p$ or to $L^{1}\left([0, T] ; B_{p, r}^{1 / p} \cap L^{\infty}\right)$ otherwise. If $f \in L^{\infty}\left([0, T] ; B_{p, r}^{s}\right) \cap C\left([0, T] ; S^{\prime}\right)$ satisfies the 1-D transport equation

$$
\begin{gathered}
f_{t}+v \cdot \nabla f=F, \\
f(t, x+1)=f(t, x), \\
\left.f\right|_{t=0}=f_{0},
\end{gathered}
$$

where $v: \mathbb{R} \times \mathbb{R}^{n} \rightarrow \mathbb{R}^{n}$ stands for a given time dependent vector field, $f_{0}: \mathbb{R}^{n} \rightarrow \mathbb{R}^{m}$ and $F: \mathbb{R} \times \mathbb{R}^{n} \rightarrow \mathbb{R}^{m}$ are known data. There exists a constant $C$ depending only on $s, p$, and $r$ such that the following statements hold.
(1) If $r=1$ or $s \neq 1+1 / p$,

$$
\begin{aligned}
\|f(t)\|_{B_{p, r}^{s}} \leq & \left\|f_{0}\right\|_{B_{p, r}^{s}} \\
& +\int_{0}^{t}\|F(\tau)\|_{B_{p, r}^{s}} d \tau+C \int_{0}^{t} V^{\prime}(\tau)\|f(\tau)\|_{B_{p, r}^{s}} d \tau,
\end{aligned}
$$

or

$$
\|f(t)\|_{B_{p, r}^{s}} \leq e^{C V(t)}\left[\left\|f_{0}\right\|_{B_{p, r}^{s}}+\int_{0}^{t} e^{-C V(\tau)}\|F(\tau)\|_{B_{p, r}^{s}} d \tau\right],
$$

where

$$
V(t)=\left\{\begin{array}{l}
\int_{0}^{t}\left\|v_{x}(\tau)\right\|_{B_{p, r}^{1 / p} \cap L^{\infty}} d \tau, \\
s<1+\frac{1}{p}, \\
\int_{0}^{t}\left\|v_{x}(\tau)\right\|_{B_{p, r}^{s-1}} d \tau, \\
s>1+\frac{1}{p} \text { or } s=1+\frac{1}{p}, r=1 .
\end{array}\right.
$$

(2) If $s \leq 1+1 / p, f_{0 x} \in L^{\infty}, f_{x} \in L^{\infty}((0, T) \times \mathbb{S}), F_{x} \in$ $L^{1}\left((0, T) ; L^{\infty}\right)$, then

$$
\begin{aligned}
\|f\|_{B_{p, r}^{s}} & +\left\|f_{x}\right\|_{L^{\infty}} \\
\leq & e^{C V(t)}\left[\left\|f_{0}\right\|_{B_{p, r}^{s}}+\left\|f_{0 x}\right\|_{L^{\infty}}\right. \\
& \left.+\int_{0}^{t} e^{-C V(\tau)}\left(\|F\|_{B_{p, r}^{s}}+\left\|F_{x}\right\|_{L^{\infty}}\right) d \tau\right],
\end{aligned}
$$

where $V(t)=\int_{0}^{t}\left\|\partial_{x} v(\tau)\right\|_{B_{p, r}^{1 / p} \cap L^{\infty}} d \tau$.

(3) If $f=v$, then for all $s>0,(23)$ holds true with $V(t)=$ $\int_{0}^{t}\left\|v_{x}(\tau)\right\|_{L^{\infty}} d \tau$.

(4) If $r<\infty$, then $f \in C\left([0, T] ; B_{p, r}^{s}\right)$. If $r=\infty$, then $f \in C\left([0, T] ; B_{p, 1}^{s^{\prime}}\right)$ for all $s^{\prime}<s$.

Lemma 10 (see [46]). Let $p, r, s, f_{0}, F$ be defined as in Lemma 9. Assume $v \in L^{\rho}\left([0, T] ; B_{\infty, \infty}^{-M}\right)$ for some $\rho>1$, $M>0 . v_{x} \in L^{1}\left([0, T] ; B_{p, r}^{s-1}\right)$ if $s>1+1 / p$ or $s=1+1 / p, r=1$; and $v_{x} \in L^{1}\left([0, T] ; B_{p, \infty}^{1 / p} \cap L^{\infty}\right)$ if $s<1+1 / p$. Then, (21) has a unique solution $f \in L^{\infty}\left([0, T] ; B_{p, r}^{s}\right) \cap\left(\cap_{s^{\prime}<s} C\left([0, T] ; B_{p, 1}^{s^{\prime}}\right)\right)$ and (23) holds true. If $r<\infty$, then $f \in C\left([0, T] ; B_{p, r}^{s}\right)$.

Lemma 11 (see [32]). Let $0<\sigma<1$. Assume $f_{0} \in$ $H^{\sigma}, F \in L^{1}\left([0, T] ; H^{\sigma}\right), v$ and $\partial_{x} v \in L^{1}\left([0, T] ; L^{\infty}\right)$. If $f \in L^{\infty}\left([0, T] ; H^{\sigma}\right) \cap C\left([0, T] ; S^{\prime}\right)$ satisfies $(21)$, then $f \in$ $C\left([0, T] ; H^{\sigma}\right)$, and there exists a constant $C$ depending only on $\sigma$ such that the statements hold:

$$
\begin{aligned}
\|f(t)\|_{H^{\sigma}} \leq & \left\|f_{0}\right\|_{H^{\sigma}}+C \int_{0}^{t}\|F(\tau)\|_{H^{\sigma}} d \tau \\
& +C \int_{0}^{t} V^{\prime}(\tau)\|f(\tau)\|_{H^{\sigma}} d \tau,
\end{aligned}
$$


or

$$
\|f(t)\|_{H^{\sigma}} \leq e^{C V(t)}\left[\left\|f_{0}\right\|_{H^{\sigma}}+\int_{0}^{t}\|F(\tau)\|_{H^{\sigma}} d \tau\right],
$$

where $V(t)=\int_{0}^{t}\left(\|v(\tau)\|_{L^{\infty}}+\left\|\partial_{x} v(\tau)\right\|_{L^{\infty}}\right) d \tau$.

\section{The Proof of Theorem 1}

We finish the proof with two subsections.

3.1. Existence of Solutions. We use a standard iterative process to construct approximate solutions to system (6).

Step 1. Starting from $u^{0}=\rho^{0}=0$, we define by induction a sequence of smooth functions $\left(u^{i}, \rho^{i}\right)_{i \in \mathbb{N}} \in C\left(\mathbb{R}^{+} ; B_{p, r}^{\infty}(\mathbb{S})\right)^{2}$ satisfying

$$
\begin{gathered}
\left(\partial_{t}+u^{i} \partial_{x}\right) u^{i+1}=F(t, x), \quad t>0, \quad x \in \mathbb{S}, \\
\left(\partial_{t}+u^{i} \partial_{x}\right) \rho^{i+1}=F_{0}(t, x), \quad t>0, \quad x \in \mathbb{S}, \\
u^{i+1}(t, x)=u^{i+1}(t, x+1), \quad \rho^{i+1}(t, x)=\rho^{i+1}(t, x+1), \\
\quad t \geq 0, \quad x \in \mathbb{S}, \\
u^{i+1}(0, x)=u_{0}^{i+1}(x)=S_{i+1} u_{0}, \\
\eta^{i+1}(0, x)=\eta_{0}^{i+1}(x)=S_{i+1} \eta_{0}, \\
x \in \mathbb{S},
\end{gathered}
$$

where $F(t, x)=P(D)\left[(3 / 2)\left(u^{i}\right)^{2}+(c / 2)\left(\rho^{i}\right)^{2}\right]-\lambda_{1} u^{i}, F_{0}(t, x)=$ $-2 \partial_{x} u^{i} \rho^{i}-\lambda \rho^{i}$.

Since all the data $S_{i+1} u_{0}, S_{i+1} \rho_{0} \in B_{p, r}^{\infty}$, Lemma 10 enables us to show that, for all $i \in \mathbb{N}$, system (28) has a global solution which belongs to $C\left(\mathbb{R}^{+} ; B_{p, r}^{\infty}(\mathbb{S})\right)^{2}$.

Step 2. Now we are in the position to prove that $\left(u^{i}, \rho^{i}\right)_{i \in \mathbb{N}}$ is uniformly bounded in $E_{p, r}^{\mathcal{s}}(T) \times E_{p, r}^{s-1}(T)$.

According to Lemma 9, for all $i \in \mathbb{N}$, one has

$$
\begin{aligned}
\left\|u^{i+1}\right\|_{B_{p, r}^{s} \leq} \leq & e^{C_{1} \int_{0}^{t}\left\|u^{i}\right\|_{B_{p, r}^{s}} d \tau} \\
& \times\left[\left\|u_{0}\right\|_{B_{p, r}^{s}}+\int_{0}^{t} e^{-C_{1} \int_{0}^{\tau}\left\|u^{i}\right\|_{B_{p, r}^{s}} d \xi}\|F(\tau)\|_{B_{p, r}^{s}} d \tau\right], \\
\left\|\rho^{i+1}\right\|_{B_{p, r}^{s-1}} \leq & e^{C_{2} \int_{0}^{t}\left\|u^{i}\right\|_{B_{p, r}^{s}} d \tau} \\
& \times\left[\left\|\rho_{0}\right\|_{B_{p, r}^{s-1}}+\int_{0}^{t} e^{-C_{2} \int_{0}^{\tau}\left\|u^{i}\right\|_{B_{p, r}^{s}}^{s} d \xi}\left\|F_{0}(\tau)\right\|_{B_{p, r}^{s-1}} d \tau\right] .
\end{aligned}
$$

We know if $\max (3 / 2,1+1 / p)<s \leq 2+1 / p$, then $B_{p, r}^{s-1}$ is an algebra. And if $s>2+1 / p$, then $B_{p, r}^{s-2}$ is an algebra. Moreover, combining (7) of Proposition 8 and

$$
\begin{aligned}
P(D) u(x) & =\sum_{\xi \in \mathbb{Z}} e^{2 \pi i x \xi} \widehat{P(D) u}(\xi) \\
& =\sum_{\xi \in \mathbb{Z}} e^{2 \pi i x \xi} \frac{2 \pi i \xi}{1+4 \pi^{2} \xi^{2}} \widehat{u}(\xi),
\end{aligned}
$$

one deduces

$$
\|P(D) u\|_{B_{p, r}^{s+1}} \leq C\|u\|_{B_{p, r}^{s}} \quad \text { if } u \in B_{p, r}^{s} .
$$

Using (6) of Proposition 8 yields

$$
\begin{gathered}
\|F(t, x)\|_{B_{p, r}^{s}} \leq C\left(\left\|u^{i}\right\|_{B_{p, r}^{s}}^{2}+\left\|\rho^{i}\right\|_{B_{p, r}^{s-1}}^{2}+\left\|u^{i}\right\|_{B_{p, r}^{s}}\right), \\
\left\|F_{0}(t, x)\right\|_{B_{p, r}^{s-1}} \leq C\left(\left\|u^{i}\right\|_{B_{p, r}^{s}}\left\|\rho^{i}\right\|_{B_{p, r}^{s-1}}+\left\|\rho^{i}\right\|_{B_{p, r}^{s-1}}\right) .
\end{gathered}
$$

Therefore, from (29) to (32), one gets

$$
\begin{aligned}
& \left\|u^{i+1}\right\|_{B_{p, r}^{s}}+\left\|\rho^{i+1}\right\|_{B_{p, r}^{s-1}} \\
& \leq C_{3} \cdot e^{C_{3} \int_{0}^{t}\left\|u^{i}\right\|_{B_{p, r}^{s}} d \tau} \\
& \times\left[\left(\left\|u_{0}\right\|_{B_{p, r}^{s}}+\left\|\rho_{0}\right\|_{B_{p, r}^{s-1}}\right)\right. \\
& +\int_{0}^{t} e^{-C_{3} \int_{0}^{\tau}\left\|u^{i}\right\|_{B_{p, r}^{s}}^{s} d \xi}\left(\left\|u^{i}\right\|_{B_{p, r}^{s}}+\left\|\rho^{i}\right\|_{B_{p, r}^{s-1}}+1\right) \\
& \left.\quad \times\left(\left\|u^{i}\right\|_{B_{p, r}^{s}}+\left\|\rho^{i}\right\|_{B_{p, r}^{s-1}}\right)\right] d \tau .
\end{aligned}
$$

Let us choose a $T>0$ such that $2 C_{3}^{2}\left(\left\|u_{0}^{i}\right\|_{B_{p, r}^{s}}+\left\|\rho_{0}^{i}\right\|_{B_{p, r}^{s-1}}+1\right) T<$ 1 and

$$
1+\left\|u^{i}\right\|_{B_{p, r}^{s}}+\left\|\rho^{i}\right\|_{B_{p, r}^{s-1}} \leq \frac{C_{3}\left(1+\left\|u_{0}\right\|_{B_{p, r}^{s}}+\left\|\rho_{0}\right\|_{B_{p, r}^{s-1}}\right)}{1-2 C_{3}^{2}\left(1+\left\|u_{0}\right\|_{B_{p, r}^{s}}+\left\|\rho_{0}\right\|_{B_{p, r}^{s-1}}\right) t} .
$$

Pluging (34) into (33) yields

$$
\begin{aligned}
1+\left\|u^{i+1}\right\|_{B_{p, r}^{s}}+\left\|\rho^{i+1}\right\|_{B_{p, r}^{s-1}} & \\
\leq & \frac{C_{3}\left(1+\left\|u_{0}\right\|_{B_{p, r}^{s}}+\left\|\rho_{0}\right\|_{B_{p, r}^{s-1}}\right)}{1-2 C_{3}^{2}\left(1+\left\|u_{0}\right\|_{B_{p, r}^{s}}+\left\|\rho_{0}\right\|_{B_{p, r}^{s-1}}\right) t} .
\end{aligned}
$$

Therefore, $\left(u^{i}, \rho^{i}\right)_{i \in \mathbb{N}}$ is uniformly bounded in $C([0, T]$; $\left.B_{p, r}^{s}(\mathbb{S})\right) \times C\left([0, T] ; B_{p, r}^{s-1}(\mathbb{S})\right)$. From Proposition 8 and the embedding properties

$$
B_{p, r}^{s} \hookrightarrow B_{p, r}^{s-1}, \quad B_{p, r}^{s-1} \hookrightarrow B_{p, r}^{s-2},
$$


one obtains

$$
\begin{aligned}
& \left\|u^{i} \partial_{x} u^{i+1}\right\|_{B_{p, r}^{s-1}} \leq\left\|u^{i}\right\|_{B_{p, r}^{s-1}}\left\|\partial_{x} u^{i+1}\right\|_{B_{p, r}^{s-1}} \leq\left\|u^{i}\right\|_{B_{p, r}^{s}}\left\|u^{i+1}\right\|_{B_{p, r}^{s}}, \\
& \left\|u^{i} \partial_{x} \rho^{i+1}\right\|_{B_{p, r}^{s-2}} \leq\left\|u^{i}\right\|_{B_{p, r}^{s-1}}\left\|\partial_{x} \rho^{i+1}\right\|_{B_{p, r}^{s-2}} \leq\left\|u^{i}\right\|_{B_{p, r}^{s}}\left\|\rho^{i+1}\right\|_{B_{p, r}^{s-1}} .
\end{aligned}
$$

Thus, we conclude that $u^{i} u_{x}^{i+1}$ and $F(t, x)$ are uniformly bounded in $C\left([0, T] ; B_{p, r}^{s-1}(\mathbb{S})\right)$. In the same way we have that $u^{i} \rho_{x}^{i+1}$ and $F_{0}(t, x)$ are uniformly bounded in $C\left([0, T] ; B_{p, r}^{s-2}(\mathbb{S})\right)$. Using (28), one obtains that $\left(\partial_{t} u^{i+1}, \partial_{t} \rho^{i+1}\right) \in C\left([0, T] ; B_{p, r}^{s-1}(\mathbb{S})\right) \times C\left([0, T] ; B_{p, r}^{s-2}(\mathbb{S})\right)$ is uniformly bounded, which yields that $\left(u^{i}, \rho^{i}\right)_{i \in \mathbb{N}}$ is uniformly bounded in $E_{p, r}^{\mathcal{s}}(T) \times E_{p, r}^{s-1}(T)$.

Step 3. We demonstrate that $\left(u^{i}, \rho^{i}\right)_{i \in \mathbb{N}}$ is a Cauchy sequence in $C\left([0, T] ; B_{p, r}^{s-1}(\mathbb{S})\right) \times C\left([0, T] ; B_{p, r}^{s-2}(\mathbb{S})\right)$.

In fact, according to (28), for all $i, j \in \mathbb{N}$, one has

$$
\begin{aligned}
& \left(\partial_{t}+u^{i+j} \partial_{x}\right)\left(u^{i+j+1}-u^{i+1}\right) \\
& =\left(u^{i}-u^{i+j}\right) \partial_{x} u^{i+1}+P(D) \\
& \quad \times\left[\frac{3}{2}\left(u^{i+j}-u^{i}\right) \times\left(u^{i+j}+u^{i}\right)+\frac{c}{2}\left(\rho^{i+j}-\rho^{i}\right)\left(\rho^{i+j}+\rho^{i}\right)\right] \\
& \quad-\lambda_{1}\left(u^{i+j}-u^{i}\right)
\end{aligned}
$$

$$
\begin{aligned}
\left(\partial_{t}+\right. & \left.u^{i+j} \partial_{x}\right)\left(\rho^{i+j+1}-\rho^{i+1}\right) \\
= & \left(u^{i}-u^{i+j}\right) \partial_{x} \rho^{i+1}-2\left(\rho^{i+j}-\rho^{i}\right) \partial_{x} u^{i} \\
& -2 \rho^{i+j} \partial_{x}\left(u^{i+j}-u^{i}\right)-\lambda\left(\rho^{i+j}-\rho^{i}\right) .
\end{aligned}
$$

(1) For the case $s \neq 2+1 / p$, firstly, we estimate the right side of (38). From (17) and (18), we obtain

$$
\begin{gathered}
\left\|\left(u^{i}-u^{i+j}\right) \partial_{x} u^{i+1}\right\|_{B_{p, r}^{s-1}} \leq C\left\|u^{i+j}-u^{i}\right\|_{B_{p, r}^{s-1}}\left\|\partial_{x} u^{i+1}\right\|_{B_{p, r}^{s-1}}, \\
\left\|P(D)\left(u^{i+j}-u^{i}\right)\left(u^{i+j}+u^{i}\right)\right\|_{B_{p, r}^{s-1}} \\
\leq C\left\|u^{i+j}-u^{i}\right\|_{B_{p, r}^{s-1}}\left\|u^{i+j}+u^{i}\right\|_{B_{p, r}^{s-1}}, \\
\left\|P(D)\left[\left(\rho^{i+j}-\rho^{i}\right)\left(\rho^{i+j}+\rho^{i}\right)\right]\right\|_{B_{p, r}^{s-1}} \\
\leq C\left\|\rho^{i+j}-\rho^{i}\right\|_{B_{p, r}^{s-2}}\left\|\rho^{i+j}+\rho^{i}\right\|_{B_{p, r}^{s-1}}, \\
\left\|\lambda_{1}\left(u^{i+j}-u^{i}\right)\right\|_{B_{p, r}^{s-1}} \leq C \lambda_{1}\left\|u^{i+j}-u^{i}\right\|_{B_{p, r}^{s-1}} .
\end{gathered}
$$

Secondly, we estimate the right side of (39). Using (18), one gets

$$
\begin{gathered}
\left\|\left(u^{i}-u^{i+j}\right) \partial_{x} \rho^{i+1}\right\|_{B_{p, r}^{s-2}} \leq C\left\|u^{i+j}-u^{i}\right\|_{B_{p, r}^{s-1}}\left\|\partial_{x} \rho^{i+1}\right\|_{B_{p, r}^{s-2}}, \\
\left\|\left(\rho^{i+j}-\rho^{i}\right) \partial_{x} u^{i}\right\|_{B_{p, r}^{s-2}} \leq C\left\|\rho^{i+j}-\rho^{i}\right\|_{B_{p, r}^{s-2}}\left\|\partial_{x} u^{i}\right\|_{B_{p, r}^{s-1}}, \\
\left\|\rho^{i+j} \partial_{x}\left(u^{i+j}-u^{i}\right)\right\|_{B_{p, r}^{s-2}} \leq C\left\|\partial_{x}\left(u^{i+j}-u^{i}\right)\right\|_{B_{p, r}^{s-2}}\left\|\rho^{i+j}\right\|_{B_{p, r}^{s-1}} .
\end{gathered}
$$

For all $t \in[0, T]$, it is deduced from Lemma 9 that

$$
\begin{aligned}
& \left\|u^{i+j+1}-u^{i+1}\right\|_{B_{p, r}^{s-1}} \\
& \leq e^{C \int_{0}^{t}\left\|u^{i+j}\right\|_{B_{p, r}^{s}} d \tau} \\
& \times\left\{\left\|u_{0}^{i+j+1}-u_{0}^{i+1}\right\|_{B_{p, r}^{s-1}}\right. \\
& +C \int_{0}^{t} e^{-C \int_{0}^{\tau}\left\|u^{i+j}\right\|_{B_{p, r}^{s}} d \xi} \\
& \times\left[\left\|\rho^{i+j}-\rho^{i}\right\|_{B_{p, r}^{s-2}}\right. \\
& \quad \times\left(\left\|\rho^{i+j}\right\|_{B_{p, r}^{s-1}}+\left\|\rho^{i}\right\|_{B_{p, r}^{s-1}}\right)+\left\|u^{i+j}-u^{i}\right\|_{B_{p, r}^{s-1}} \\
& \quad \times\left(\left\|u^{i}\right\|_{B_{p, r}^{s}}+\left\|u^{i+j}\right\|_{B_{p, r}^{s}}\right. \\
& \left.\left.\left.\quad+\left\|u^{i+1}\right\|_{B_{p, r}^{s}}+\lambda_{1}\right)\right] d \tau\right\},
\end{aligned}
$$

$$
\left\|\rho^{i+j+1}-\rho^{i+1}\right\|_{B_{p, r}^{s-2}}
$$

$$
\begin{gathered}
\leq e^{C \int_{0}^{t}\left\|u^{i+j}\right\|_{B_{p, r}^{s}} d \tau} \\
\times\left\{\left\|\rho_{0}^{i+j+1}-\rho_{0}^{i+1}\right\|_{B_{p, r}^{s-2}}\right. \\
+C \int_{0}^{t} e^{-C \int_{0}^{\tau}\left\|u^{i+j}\right\|_{B_{p, r}^{s}} d \xi} \\
\times\left[\left\|u^{i+j}-u^{i}\right\|_{B_{p, r}^{s-1}}\right. \\
\quad \times\left(\left\|\rho^{i+1}\right\|_{B_{p, r}^{s-1}}+\left\|\rho^{i+j}\right\|_{B_{p, r}^{s-1}}\right)+\left\|\rho^{i+j}-\rho^{i}\right\|_{B_{p, r}^{s-2}} \\
\left.\left.\quad \times\left(\left\|u^{i}\right\|_{B_{p, r}^{s}}+\lambda\right)\right] d \tau\right\} .
\end{gathered}
$$


Since $\left(u^{i}, \rho^{i}\right)_{i \in \mathbb{N}}$ is uniformly bounded in $E_{p, r}^{s}(T) \times E_{p, r}^{s-1}(T)$ and

$$
u_{0}^{i+j+1}-u_{0}^{i+1}=\sum_{q=i+1}^{i+j} \Delta_{q} u_{0}, \quad \rho_{0}^{i+j+1}-\rho_{0}^{i+1}=\sum_{q=i+1}^{i+j} \Delta_{q} \rho_{0},
$$

there exists a constant $C_{T}$ independent of $i, j$ such that for all $t \in[0, T]$

$$
\begin{aligned}
& \left\|u^{i+j+1}-u^{i+1}\right\|_{B_{p, r}^{s-1}}+\left\|\rho^{i+j+1}-\rho^{i+1}\right\|_{B_{p, r}^{s-2}} \\
& \quad \leq C_{T}\left[2^{-i}+\int_{0}^{t}\left(\left\|u^{i+j}-u^{i}\right\|_{B_{p, r}^{s-1}}+\left\|\rho^{i+j}-\rho^{i}\right\|_{B_{p, r}^{s-2}}\right) d \tau\right] .
\end{aligned}
$$

By induction, one obtains

$$
\begin{aligned}
& \left\|u^{i+j+1}-u^{i+1}\right\|_{L_{T}^{\infty}\left(B_{p, r}^{s-1}\right)}+\left\|\rho^{i+j+1}-\rho^{i+1}\right\|_{L_{T}^{\infty}\left(B_{p, r}^{s-2}\right)} \\
& \leq \frac{\left(C_{T} T\right)^{i+1}}{(i+1) !}\left[\left\|u^{j}\right\|_{L^{\infty}\left([0, T] ; B_{p, r}^{s-1}\right)}+\left\|\rho^{j}\right\|_{L^{\infty}\left([0, T] ; B_{p, r}^{s-2}\right)}\right] \\
& \quad+C_{T} \sum_{l=0}^{i} 2^{-(i-l)} \frac{\left(C_{T} T\right)^{l}}{l !} .
\end{aligned}
$$

Since $\left\|u^{j}\right\|_{L_{T}^{\infty}\left(B_{p, r}^{s}\right)},\left\|\rho^{j}\right\|_{L_{T}^{\infty}\left(B_{p, r}^{s, 1}\right)}$ are bounded independent of $j$, there exists a new constant $C_{T_{1}}$ such that

$$
\left\|u^{i+j+1}-u^{i+1}\right\|_{L_{T}^{\infty}\left(B_{p, r}^{s-1}\right)}+\left\|\rho^{i+j+1}-\rho^{i+1}\right\|_{L_{T}^{\infty}\left(B_{p, r}^{s-2}\right)} \leq C_{T_{1}} 2^{-i} .
$$

Consequently, $\left(u^{i}, \rho^{i}\right)_{i \in \mathbb{N}}$ is a Cauchy sequence in $C([0, T]$; $\left.B_{p, r}^{s-1}(\mathbb{S})\right) \times C\left([0, T] ; B_{p, r}^{s-2}(\mathbb{S})\right)$.

(2) For the case $s=2+1 / p$, using (4) of Proposition 8, one has

$$
\begin{aligned}
& \left\|u^{i+j+1}-u^{i+1}\right\|_{L_{T}^{\infty}\left(B_{p, r}^{s-1}\right)} \\
& \quad=\left\|u^{i+j+1}-u^{i+1}\right\|_{L_{T}^{\infty}\left(B_{p, r}^{1+1 / p}\right)} \\
& \leq\left\|u^{i+j+1}-u^{i+1}\right\|_{L_{T}^{\infty}\left(B_{p, r}^{s 1}\right)}^{\theta}\left\|u^{i+j+1}-u^{i+1}\right\|_{L_{T}^{\infty}\left(B_{p, r}^{s, r}\right)}^{1-\theta} \\
& \leq\left\|u^{i+j+1}-u^{i+1}\right\|_{B_{p, r}^{1+1 / p}}^{\theta}\left[\left\|u^{i+j+1}\right\|_{B_{p, r}^{2+1 / p}}+\left\|u^{i+1}\right\|_{B_{p, r}^{2+1 / p}}\right]^{1-\theta} \\
& \leq\left(C_{T}^{\prime}\right)^{\theta} 2^{-\theta i}\left[\left\|u^{i+j+1}\right\|_{B_{p, r}^{2+1 / p}}+\left\|u^{i+1}\right\|_{B_{p, r}^{2+1 / p}}\right]^{1-\theta},
\end{aligned}
$$

where $s_{1} \in(\max (1+1 / p, 3 / 2)-1,1+1 / p), s_{2} \in(1+1 / p, 2+$ $1 / p)$, and

$$
\begin{aligned}
& \left\|\rho^{i+j+1}-\rho^{i+1}\right\|_{L_{T}^{\infty}\left(B_{p, r}^{s, 2}\right)} \\
& \quad=\left\|\rho^{i+j+1}-\rho^{i+1}\right\|_{L_{T}^{\infty}\left(B_{p, r}^{1 / p}\right)} \\
& \leq\left\|\rho^{i+j+1}-\rho^{i+1}\right\|_{L_{T}^{\infty}\left(B_{p, r}^{s_{3}}\right)}^{\theta_{1}}\left\|\rho^{i+j+1}-\rho^{i+1}\right\|_{L_{T}^{\infty}\left(B_{p, r}^{s_{4}}\right)}^{1-\theta_{1}} \\
& \leq\left\|\rho^{i+j+1}-\rho^{i+1}\right\|_{B_{p, r}^{1 / p}}^{\theta_{1}^{1 / p}}\left[\left\|\rho^{i+j+1}\right\|_{B_{p, r}^{1+1 / p}}+\left\|\rho^{i+1}\right\|_{B_{p, r}^{1+1 / p}}\right]^{1-\theta_{1}} \\
& \leq\left(C_{T}^{\prime}\right)^{\theta_{1}} 2^{-\theta_{1} i}\left[\left\|\rho^{i+j+1}\right\|_{B_{p, r}^{1+1 / p}}+\left\|\rho^{i+1}\right\|_{B_{p, r}^{1+1 / p}}\right]^{1-\theta_{1}},
\end{aligned}
$$

where $s_{3} \in(\max (1+1 / p, 3 / 2)-2,1 / p), s_{4} \in(1 / p, 1+1 / p)$.

One deduces that $\left(u^{i}, \rho^{i}\right)_{i \in \mathbb{N}}$ is a Cauchy sequence in $C\left([0, T] ; B_{p, r}^{s-1}(\mathbb{S})\right) \times C\left([0, T] ; B_{p, r}^{s-2}(\mathbb{S})\right)$ for the critical case.

Step 4. We end the proof of existence of solutions.

Firstly, since $\left(u^{i}, \rho^{i}\right)_{i \in \mathbb{N}}$ is uniformly bounded in $L^{\infty}\left([0, T] ; B_{p, r}^{s}(\mathbb{S})\right) \times L^{\infty}\left([0, T] ; B_{p, r}^{s-1}(\mathbb{S})\right)$, according to Fatou's Lemma in Besov space, it guarantees that $(u, \rho)$ belongs to $L^{\infty}\left([0, T] ; B_{p, r}^{s}(\mathbb{S})\right) \times L^{\infty}\left([0, T] ; B_{p, r}^{s-1}(\mathbb{S})\right)$.

Secondly, since $\left(u^{i}, \rho^{i}\right)_{i \in \mathbb{N}}$ is a Cauchy sequence in $C\left([0, T] ; B_{p, r}^{s-1}(\mathbb{S})\right) \times C\left([0, T] ; B_{p, r}^{s-2}(\mathbb{S})\right)$, it converges to limit function $(u, \rho) \in C\left([0, T] ; B_{p, r}^{s-1}(\mathbb{S})\right) \times C\left([0, T] ; B_{p, r}^{s-2}(\mathbb{S})\right)$. An interpolation argument insures that the convergence holds in $C\left([0, T] ; B_{p, r}^{s^{\prime}}(\mathbb{S})\right) \times C\left([0, T] ; B_{p, r}^{s^{\prime}-1}(\mathbb{S})\right)$ for any $s^{\prime}<s$. Taking the limit in $(28)$ derives that $(u, \rho)$ is indeed a solution to (6). Thanks to the fact $(u, \rho) \in L^{\infty}\left([0, T] ; B_{p, r}^{s}(\mathbb{S})\right) \times$ $L^{\infty}\left([0, T] ; B_{p, r}^{s-1}(\mathbb{S})\right)$, we know that the right side of the first equation in (6) belongs to $L^{\infty}\left([0, T] ; B_{p, r}^{s}(\mathbb{S})\right)$, and the right side of the second equation in (6) belongs to $L^{\infty}\left([0, T] ; B_{p, r}^{s-1}(\mathbb{S})\right)$. For the case $r<\infty$, applying Lemma 9 derives $(u, \rho) \in C\left([0, T] ; B_{p, r}^{s^{\prime}}(\mathbb{S})\right) \times C\left([0, T] ; B_{p, r}^{s^{\prime}-1}(\mathbb{S})\right)$ for any $s^{\prime}<s$.

Finally, from (6), one has $\left(u_{t}, \rho_{t}\right) \in C\left([0, T] ; B_{p, r}^{s-1}(\mathbb{S})\right) \times$ $C\left([0, T] ; B_{p, r}^{s-2}(\mathbb{S})\right)$ if $r<\infty$, and in $L^{\infty}\left([0, T] ; B_{p, r}^{s-1}(\mathbb{S})\right) \times$ $L^{\infty}\left([0, T] ; B_{p, r}^{s-2}(\mathbb{S})\right)$ otherwise. Thus $(u, \rho) \in E_{p, r}^{s}(T) \times$ $E_{p, r}^{s-1}(T)$. A standard use of a sequence of viscosity approximate solutions $\left(u_{\varepsilon}, \eta_{\varepsilon}\right)_{\varepsilon>0}$ for (6) which converges uniformly in $C\left([0, T] ; B_{p, r}^{s}(\mathbb{S})\right) \cap C^{1}\left([0, T] ; B_{p, r}^{s-1}(\mathbb{S})\right) \times C\left([0, T] ; B_{p, r}^{s-1}(\mathbb{S})\right) \cap$ $C^{1}\left([0, T] ; B_{p, r}^{s-2}(\mathbb{S})\right)$ gives the continuity of solution $(u, \rho) \in$ $E_{p, r}^{s}(T) \times E_{p, r}^{s-1}(T)$.

\subsection{Uniqueness and Continuity with Initial Data}

Lemma 12. Let $1 \leq p, r \leq \infty, s>\max (1+1 / p, 3 / 2)$. Assume that $\left(u^{1}, \rho^{1}\right)$ and $\left(u^{2}, \rho^{2}\right)$ are two given solutions to the Cauchy problem (6) with initial data $\left(u_{0}^{1}, \rho_{0}^{1}\right),\left(u_{0}^{2}, \rho_{0}^{2}\right) \in$ $B_{p, r}^{s}(\mathbb{S}) \times B_{p, r}^{s-1}(\mathbb{S})$ satisfying $u^{1}, u^{2} \in L^{\infty}\left([0, T] ; B_{p, r}^{s}(\mathbb{S})\right) \cap$ 
$C\left([0, T] ; B_{p, r}^{s-1}(\mathbb{S})\right)$, and $\rho^{1}, \rho^{2} \in L^{\infty}\left([0, T] ; B_{p, r}^{s-1}(\mathbb{S})\right) \cap C([0, T]$; $\left.B_{p, r}^{s-2}(\mathbb{S})\right)$. Then, for all $t \in[0, T]$,

$$
\begin{aligned}
\| u^{1}- & u^{2}\left\|_{B_{p, r}^{s-1}}+\right\| \rho^{1}-\rho^{2} \|_{B_{p, r}^{s s-2}} \\
\leq & \left(\left\|u_{0}^{1}-u_{0}^{2}\right\|_{B_{p, r}^{s-1}}+\left\|\rho_{0}^{1}-\rho_{0}^{2}\right\|_{B_{p, r}^{s-2}}\right) \\
& \quad \times e^{C \int_{0}^{t}\left[\left(\left\|u^{1}\right\|_{B_{p, r}^{s}}^{s}+\left\|u^{2}\right\|_{B_{p, r}^{s}}^{s}+1\right)+\left(\left\|\rho^{1}\right\|_{B_{p, r}^{s-1}}+\left\|\rho^{2}\right\|_{B_{p, r}^{s-1}}\right)\right] d \tau} .
\end{aligned}
$$

Proof. Let $u^{12}=u^{2}-u^{1}, \rho^{12}=\rho^{2}-\rho^{1}$; then

$$
\begin{aligned}
& u^{12} \in L^{\infty}\left([0, T] ; B_{p, r}^{s}(\mathbb{S})\right) \cap C\left([0, T] ; B_{p, r}^{s-1}(\mathbb{S})\right), \\
& \rho^{12} \in L^{\infty}\left([0, T] ; B_{p, r}^{s-1}(\mathbb{S})\right) \cap C\left([0, T] ; B_{p, r}^{s-2}(\mathbb{S})\right),
\end{aligned}
$$

which derives that $\left(u^{12}, \rho^{12}\right) \in C\left([0, T] ; B_{p, r}^{s-1}(\mathbb{S})\right) \times C([0, T]$; $\left.B_{p, r}^{s-2}(\mathbb{S})\right)$, and $\left(u^{12}, \rho^{12}\right)$ satisfies the transport equation

$$
\begin{aligned}
& \partial_{t} u^{12}+u^{1} \partial_{x} u^{12}=-u^{12} \partial_{x} u^{2}+F_{1}(t, x), \\
& t>0, \quad x \in \mathbb{S}, \\
& \partial_{t} \rho^{12}+u^{1} \partial_{x} \rho^{12}=F_{2}(t, x), \\
& t>0, \quad x \in \mathbb{S}, \\
& \begin{array}{r}
u^{12}(t, x)=u^{12}(t, x+1), \\
\rho^{12}(t, x)=\rho^{12}(t, x+1), \\
t \geq 0, \quad x \in \mathbb{S},
\end{array} \\
& u^{12}(0, x)=u_{0}^{12}=u_{0}^{2}-u_{0}^{1}, \quad \eta^{12}(0, x)=\eta_{0}^{12}=\eta_{0}^{2}-\eta_{0}^{1}, \\
& x \in \mathbb{S},
\end{aligned}
$$

where

$$
\begin{aligned}
F_{1}(t, x)= & P(D)\left[\frac{3}{2} u^{12}\left(u^{1}+u^{2}\right)+\frac{c}{2} \rho^{12}\left(\rho^{1}+\rho^{2}\right)\right] \\
& -\lambda_{1} u^{12}, \\
F_{2}(t, x)= & -u^{12} \partial_{x} \rho^{2}-2\left(\partial_{x} u^{1} \rho^{12}+\partial_{x} u^{12} \rho^{2}\right)-\lambda \rho^{12} .
\end{aligned}
$$

According to Lemma 9, one deduces

$$
\begin{aligned}
& e^{-C \int_{0}^{t}\left\|\partial_{x} u^{1}\right\|_{B_{p, r}^{s-r}}^{s} d \tau}\left\|u^{12}\right\|_{B_{p, r}^{s-1}} \\
& \leq\left\|u_{0}^{12}\right\|_{B_{p, r}^{s-1}}+C \int_{0}^{t} e^{-C \int_{0}^{\tau}\left\|\partial_{x} u^{1}\right\|_{B_{p, r}^{s-1}}^{s-1} d \xi} \\
& \quad \times\left(\left\|u^{12} \partial_{x} u^{2}\right\|_{B_{p, r}^{s-1}}+\left\|F_{1}\right\|_{B_{p, r}^{s-1}}\right) d \tau \\
& e^{-C \int_{0}^{t}\left\|\partial_{x} u^{1}\right\|_{B_{p, r}^{s-1}} d \tau}\left\|\rho^{12}\right\|_{B_{p, r}^{s-2}} \\
& \leq\left\|\rho_{0}^{12}\right\|_{B_{p, r}^{s-2}}+C \int_{0}^{t} e^{-C \int_{0}^{\tau}\left\|\partial_{x} u^{1}\right\|_{B_{p, r}^{s-1}} d \xi}\left\|F_{2}\right\|_{B_{p, r}^{s-2}} d \tau
\end{aligned}
$$

Similar to the arguments in Step 3 in Section 3.1, one derives

$$
\begin{aligned}
& e^{-C \int_{0}^{t}\left\|\partial_{x} u^{1}\right\|_{B_{p, r}^{s-1}} d \tau}\left(\left\|u^{12}\right\|_{B_{p, r}^{s-1}}+\left\|\rho^{12}\right\|_{B_{p, r}^{s-2}}\right) \\
& \leq\left(\left\|u_{0}^{12}\right\|_{B_{p, r}^{s-1}}+\left\|\rho_{0}^{12}\right\|_{B_{p, r}^{s-2}}\right) \\
& +C \int_{0}^{t} e^{-C \int_{0}^{\tau}\left\|\partial_{x} u^{1}\right\|_{B_{p, r}^{s-1}} d \xi}\left(\left\|u^{12}\right\|_{B_{p, r}^{s-1}}+\left\|\rho^{12}\right\|_{B_{p, r}^{s-2}}\right) \\
& \times\left(1+\left\|u^{1}\right\|_{B_{p, r}^{s}}+\left\|u^{2}\right\|_{B_{p, r}^{s}}+\left\|\rho^{1}\right\|_{B_{p, r}^{s s-1}}+\left\|\rho^{2}\right\|_{B_{p, r}^{s-1}}\right) d \tau .
\end{aligned}
$$

Applying Gronwall's inequality completes the proof of Lemma 12.

Remark 13. For the critical case $s=2+1 / p$, the proof is similar to Step 3 in Section 3.1.

Remark 14. Note that, for every $s \in \mathbb{R}, B_{2,2}^{s}=H^{s}$. The existence time of system (1) may be chosen independently of $s$ in the following sense [50]. If $(u, \rho) \in C\left([0, T] ; H^{s}\right) \cap$ $C^{1}\left([0, T] ; H^{s-1}\right) \times C\left([0, T] ; H^{s-1}\right) \cap C^{1}\left([0, T] ; H^{s-2}\right)$ is a solution to system (1) with initial data $\left(u_{0}, \rho_{0}\right) \in H^{r} \times H^{r-1}$ for some $r>3 / 2, r \neq s$, then $(u, \rho) \in C\left([0, T] ; H^{r}\right) \cap$ $C^{1}\left([0, T] ; H^{r-1}\right) \times C\left([0, T] ; H^{r-1}\right) \cap C^{1}\left([0, T] ; H^{r-2}\right)$ with the same time $T$. In particular, if $(u, \rho) \in H^{\infty} \times H^{\infty}$, then $(u, \rho) \in$ $C\left([0, T] ; H^{\infty}\right) \times C\left([0, T] ; H^{\infty}\right)$.

\section{Wave-Breaking Phenomena}

This section is devoted to investigating conditions of wave breaking mechanisms of strong solutions to system (1). Using Theorem 1 and a simple density argument, we deduce that the desired results are valid for $s \geq 3$. Here we take $s=3$ in the proof for simplicity. We begin with three lemmas.

Lemma 15 (see [51]). Let $T>0$ and $u \in C^{1}\left([0, T] ; H^{2}(\mathbb{S})\right)$. Then for all $t \in[0, T]$ there exists at least one point $\xi(t) \in \mathbb{S}$, such that

$$
m(t)=\inf _{x \in \mathbb{S}} u_{x}(t, x)=u_{x}(t, \xi(t)) .
$$

The function $m(t)$ is absolutely continuous on $[0, T]$ with

$$
\frac{d}{d t} m(t)=u_{x t}(t, \xi(t)) \quad \text { a.e. on }[0, T] .
$$

We consider the trajectory equation

$$
\begin{gathered}
\frac{d}{d t} q(t, x)=u(t, q(t, x)), \quad t \in[0, T], \\
q(0, x)=x, \quad x \in \mathbb{S},
\end{gathered}
$$

where $u$ denotes the first component of solution $(u, \rho)$ to system (1). 
Lemma 16 (see [52]). Let $u \in C\left([0, T] ; H^{s}(\mathbb{S})\right) \cap C^{1}([0, T]$; $H^{s-1}(\mathbb{S})$ ) with $s \geq 2$. Then (59) has a unique solution $q \in$ $C^{1}([0, T] \times \mathbb{S}, \mathbb{S})$. Moreover, the map $q(t, \cdot)$ is an increasing diffeomorphism of $\mathbb{S}$ for all $t \in[0, T]$ and

$$
q_{x}(t, x)=e^{\int_{0}^{t} u_{x}(\tau, q(\tau, x)) d \tau}>0, \quad \forall(t, x) \in[0, T] \times \mathbb{S} .
$$

Lemma 17. Let $\left(u_{0}, \rho_{0}\right) \in H^{s}(\mathbb{S}) \times H^{s-1}(\mathbb{S})$ with $s>3 / 2$ and $T>0$ is the maximal existence time of corresponding solution $(u, \rho)$ to $(6)$. Then for all $(t, x) \in[0, T] \times \mathbb{S}$

$$
\rho(t, q(t, x)) q_{x}^{2}(t, x)=\rho_{0}(x) e^{-\lambda t} .
$$

Moreover, if there exists $M>0$ such that $u_{x}(t, x) \geq-M$ for all $(t, x) \in[0, T] \times \mathbb{S}$, then for all $t \in[0, T]$,

$$
\begin{gathered}
\|\rho(t, \cdot)\|_{L^{\infty}} \leq e^{(2 M-\lambda) t}\left\|\rho_{0}\right\|_{H^{s-1}}, \\
\|\rho(t, \cdot)\|_{L^{2}} \leq e^{(2 M-\lambda) t}\left\|\rho_{0}\right\|_{H^{s-1}} .
\end{gathered}
$$

Proof of Lemma 17. Differentiating the left side of (61) with respect to $t$, using (59) and the second equation in (1), we obtain

$$
\begin{aligned}
\frac{d}{d t}[ & \left.\rho(t, q(t, x)) q_{x}^{2}(t, x)\right] \\
= & \left(\rho_{t}(t, q)+\rho_{x}(t, q) q_{t}(t, x)\right) q_{x}^{2}(t, x) \\
& +2 \rho(t, q) q_{x}(t, x) q_{x t}(t, x) \\
= & {\left[\rho_{t}(t, q)+\rho_{x}(t, q) u(t, q)+2 \rho(t, q) u_{x}(t, q)\right] q_{x}^{2}(t, x) } \\
= & -\lambda \rho(t, q(t, x)) q_{x}^{2}(t, x) .
\end{aligned}
$$

Applying Gronwall's inequality and (59) yields (61).

From Lemma 16, (61) and the assumption in Lemma 17, one deduces

$$
\begin{aligned}
& \|\rho(t, \cdot)\|_{L^{\infty}} \\
& \quad=\|\rho(t, q(t, \cdot))\|_{L^{\infty}}=\left\|e^{-\lambda t} e^{-2 \int_{0}^{t} u_{x}(\tau \cdot) d \tau} \rho_{0}(\cdot)\right\|_{L^{\infty}} \\
& \leq e^{(2 M-\lambda) t}\left\|\rho_{0}(\cdot)\right\|_{L^{\infty}}, \\
& \int_{\mathbb{R}}|\rho(t, x)|^{2} d x \\
& =\int_{\mathbb{R}}|\rho(t, q(t, x))|^{2} q_{x}(t, x) d x \\
& =\int_{\mathbb{R}}\left|\rho_{0}(x) e^{-\lambda t}\right|^{2} q_{x}^{-3}(t, x) d x \\
& \leq e^{(3 M-2 \lambda) t} \int_{\mathbb{R}}\left|\rho_{0}(x)\right|^{2} d x, \quad \forall t \in[0, T] .
\end{aligned}
$$

This completes the proof of Lemma 17.

In what follows we derive the estimates for $\|u(t)\|_{L^{\infty}}$.
Lemma 18. Let $\left(u_{0}, \rho_{0}\right) \in H^{s}(\mathbb{S}) \times H^{s-1}(\mathbb{S})$ with $s>3 / 2$ and $T$ is the maximal existence time of corresponding solution $(u, \rho)$ to system (1). Assume that there exists $M>0$ such that $\|\rho(t, \cdot)\|_{L^{\infty}} \leq e^{(2 M-\lambda) t}\left\|\rho_{0}\right\|_{H^{s-1}},\|\rho(t, \cdot)\|_{L^{2}} \leq e^{(2 M-\lambda) t}\left\|\rho_{0}\right\|_{H^{s-1}}$ for all $t \in[0, T]$. Then for all $t \in[0, T]$, we have

$$
\begin{aligned}
& \|u(t)\|_{L^{2}}^{2} \\
& \leq\left[4\left\|u_{0}\right\|_{L^{2}}^{2}+2|c| T e^{\left(8 M+4 \lambda+2 \lambda_{1}\right) T}\left\|\rho_{0}\right\|_{H^{s-1}}^{4}\right] e^{2|c| t e^{2 \lambda_{1} T}} \\
& =H(t) \\
& \quad\|u(t)\|_{L^{\infty}} \leq\left\|u_{0}(x)\right\|_{L^{\infty}}+e^{\lambda_{1} t} t P(t)
\end{aligned}
$$

where $P(t)=(3 / 4) H(t)+(1 / 4)|c|\left(e^{2 M t}\left\|\rho_{0}\right\|_{H^{s-1}}\right)^{2}$.

Proof of Lemma 18. As mentioned before, here we assume $s=$ 3 to prove Lemma 18. Let $m=\left(1-\partial_{x}^{2}\right) u, w=\left(4-\partial_{x}^{2}\right)^{-1} u$. Then we rewrite the first equation in (1) as

$$
m_{t}+3 m u_{x}+m_{x} u+\lambda_{1} m+c \rho \rho_{x}=0
$$

Noting $\left(\widehat{m}_{t}, \widehat{w}\right)=\left(\widehat{m}, \widehat{w}_{t}\right)$ or $\int_{\mathbb{S}} m_{t} w d x=\int_{\mathbb{S}} m w_{t} d x$, one has

$$
\begin{aligned}
& \frac{1}{2} \frac{d}{d t} \int_{\mathbb{S}} m w d x \\
& =\frac{1}{2} \int_{\mathbb{S}} m_{t} w d x+\frac{1}{2} \int_{\mathbb{S}} m w_{t} d x=\int_{\mathbb{S}} m_{t} w d x \\
& =-3 \int_{\mathbb{S}} w m u_{x} d x-\int_{\mathbb{S}} w m_{x} u d x \\
& \quad-c \int_{\mathbb{S}} w \rho \rho_{x} d x-\lambda_{1} \int_{\mathbb{S}} w m d x \\
& =-\int_{\mathbb{S}} w(m u)_{x} d x-2 \int_{\mathbb{S}} w m u_{x} d x \\
& \quad+\frac{c}{2} \int_{\mathbb{S}} w_{x} \rho^{2} d x-\lambda_{1} \int_{\mathbb{S}} w m d x \\
& \int_{\mathbb{S}} w(m u)_{x} d x=-\int_{\mathbb{S}} w_{x} m u d x \\
& \quad=\int_{\mathbb{S}} w_{x} u^{2} d x-\int_{\mathbb{S}} w_{x} u_{x}^{2} d x \\
& 2 \int_{\mathbb{S}} w m u_{x} d x=-\int_{\mathbb{S}} w_{x} u^{2} d x+\int_{\mathbb{S}} w_{x} u_{x}^{2} d x
\end{aligned}
$$

Combining the above three equalities, one derives

$$
\frac{d}{d t} \int_{\mathbb{S}} m w d x=-2 \lambda_{1} \int_{\mathbb{S}} m w d x+c \int_{\mathbb{S}} w_{x} \rho^{2} d x
$$


Using Gronwall's inequality, we have

$$
\begin{aligned}
& \int_{\mathbb{S}} m w d x \\
& \quad=e^{-2 \lambda_{1} t}\left[\int_{\mathbb{S}} m_{0} w_{0} d x+c \int_{0}^{t} e^{2 \lambda_{1} \tau} \int_{\mathbb{S}} w_{x} \rho^{2} d x d \tau\right] \\
& \quad \leq \int_{\mathbb{S}} m_{0} w_{0} d x+|c| e^{2 \lambda_{1} t} \int_{0}^{t} \int_{\mathbb{S}} w_{x} \rho^{2} d x d \tau
\end{aligned}
$$

Thus

$$
\begin{aligned}
& \|u(t)\|_{L^{2}}^{2} \\
& =\|\widehat{u}(t)\|_{L^{2}}^{2} \leq 4 \int_{\mathbb{S}} \frac{1+|2 \pi \xi|^{2}}{4+|2 \pi \xi|^{2}}|\widehat{u}(t, \xi)|^{2} d \xi=4(\widehat{m}(t), \widehat{w}(t)) \\
& =4(m(t), w(t))=4\left(m_{0}, w_{0}\right)+4|c| e^{2 \lambda_{1} t} \int_{0}^{t} \int_{\mathbb{S}} w_{x} \rho^{2} d x d \tau \\
& \leq 4\left\|u_{0}\right\|_{L^{2}}^{2}+4|c| e^{2 \lambda_{1} t} \int_{0}^{t} \int_{\mathbb{S}} w_{x} \rho^{2} d x d \tau .
\end{aligned}
$$

Noting

$$
\left\|w_{x}(t)\right\|_{L^{2}}^{2}=\left\|\partial_{x}\left(4-\partial_{x}^{2}\right)^{-1} u(t)\right\|_{L^{2}}^{2} \leq\|u(t)\|_{L^{2}}^{2}
$$

and using the assumption in Lemma 18, we obtain

$$
\|\rho(t, \cdot)\|_{L^{4}}^{4}=\|\rho(t, \cdot)\|_{L^{\infty}}^{2}\|\rho(t, \cdot)\|_{L^{2}}^{2} \leq\left[e^{(2 M-\lambda) t}\left\|\rho_{0}\right\|_{H^{s-1}}\right]^{4} .
$$

Hence

$$
\begin{aligned}
& \|u(t)\|_{L^{2}}^{2} \\
& \leq 4\left\|u_{0}\right\|_{L^{2}}^{2}+2|c| e^{2 \lambda_{1} t} \int_{0}^{t}\left[\left\|w_{x}(\tau, \cdot)\right\|_{L^{2}}^{2}+\|\rho(\tau, \cdot)\|_{L^{4}}^{4}\right] d \tau \\
& \leq 4\left\|u_{0}\right\|_{L^{2}}^{2}+2|c| t e^{\left(8 M+4 \lambda+2 \lambda_{1}\right) t}\left\|\rho_{0}\right\|_{H^{s-1}}^{4} \\
& \quad+2|c| e^{2 \lambda_{1} t} \int_{0}^{t}\|u(\tau)\|_{L^{2}}^{2} d \tau \\
& \leq 4\left\|u_{0}\right\|_{L^{2}}^{2}+2|c| T e^{\left(8 M+4 \lambda+2 \lambda_{1}\right) T}\left\|\rho_{0}\right\|_{H^{s-1}}^{4} \\
& \quad+2|c| e^{2 \lambda_{1} T} \int_{0}^{t}\|u(\tau)\|_{L^{2}}^{2} d \tau .
\end{aligned}
$$

Applying Gronwall's inequality yields (65).

Now we present the proof of (66). Note that, for all $x \in \mathbb{S}$, if $g(x)=\cosh (x-[x]-1 / 2) / 2 \sinh (1 / 2)$, where $[x]$ denotes the integer part of $x$, then $\left(1-\partial_{x}^{2}\right)^{-1} f=g *$ $f$ for all $f \in L^{2}(\mathbb{S})$. It follows from some calculations that $g(x)$ is continuous and decreasing on interval $[0,1 / 2]$ and increasing on interval $[1 / 2,1]: g(1 / 2)=1 / 2 \sinh (1 / 2)$, $g(0)=g(1)=\cosh (1 / 2) / 2 \sinh (1 / 2), 1 / 2 \sinh (1 / 2) \leq g(x) \leq$ $\cosh (1 / 2) / 2 \sinh (1 / 2)=c_{0}$, and $\left\|\partial_{x} g\right\|_{L^{\infty}} \leq 1 / 2$. Applying Young's inequality, one has

$$
\begin{aligned}
\left\|-\partial_{x} g *\left(\frac{3}{2} u^{2}+\frac{1}{2} c \rho^{2}\right)\right\|_{L^{\infty}} & \leq\left\|-\partial_{x} g\right\|_{L^{\infty}}\left\|\frac{3}{2} u^{2}+\frac{1}{2} c \rho^{2}\right\|_{L^{1}} \\
& \leq \frac{3}{4}\|u\|_{L^{2}}^{2}+\frac{|c|}{4}\|\rho\|_{L^{2}}^{2} .
\end{aligned}
$$

Using (59), we obtain

$$
\begin{aligned}
\frac{d u(t, q(t, x))}{d t} & =u_{t}(t, q(t, x))+u_{x}(t, q(t, x)) q_{t}(t, x) \\
& =\left(u_{t}+u u_{x}\right)(t, q(t, x)) .
\end{aligned}
$$

For the first equation in system (6), using (65) and the facts above, one derives

$$
-P(t) \leq \frac{d u(t, q(t, x))}{d t}+\lambda_{1} u(t, q(t, x)) \leq P(t) .
$$

It follows from Gronwall's inequality that

$$
|u(t, q(t, x))| \leq\left\|u_{0}(x)\right\|_{L^{\infty}}+e^{\lambda_{1} t} t P(t) .
$$

From Lemma 16, we obtain (66).

Lemma 19. Let $\left(u_{0}, \rho_{0}\right) \in H^{s}(\mathbb{S}) \times H^{s-1}(\mathbb{S})$ with $s>3 / 2$ and $T$ is the maximal existence time of corresponding solution $(u, \rho)$ to system (6). If $\partial_{x} u \in L^{1}\left([0, T] ; L^{\infty}\right)$, then for all $t \in[0, T]$, we have

$\|u(t)\|_{L^{\infty}}$

$$
\leq\left\|u_{0}(x)\right\|_{L^{\infty}}
$$

$$
\begin{aligned}
& +e^{\lambda_{1} t} t\left[\frac{3}{4} H_{1}(t)+\frac{|c|}{4}\left(e^{2 \int_{0}^{t}\left\|\partial_{x} u(\tau)\right\|_{L^{\infty}} d \tau}\left\|\rho_{0}\right\|_{H^{s-1}}\right)^{2}\right] \\
= & L(t),
\end{aligned}
$$

where

$$
\begin{aligned}
& H_{1}(t) \\
& =\left[4\left\|u_{0}\right\|_{L^{2}}^{2}+2|c| T e^{\left(8 \int_{0}^{T}\left\|\partial_{x} u(\tau)\right\|_{L^{\infty}} d \tau\right)+\left(4 \lambda+2 \lambda_{1}\right) T}\left\|\rho_{0}\right\|_{H^{s-1}}^{4}\right] \\
& \quad \times e^{2 e^{2 \lambda_{1} T}|c| t} .
\end{aligned}
$$

Proof of Lemma 19. It follows from the proof of Lemma 17 that

$$
\begin{aligned}
\|\rho(t, \cdot)\|_{L^{\infty}},\|\rho(t, \cdot)\|_{L^{2}} & \leq e^{-\lambda t+2 \int_{0}^{t}\left\|\partial_{x} u(\tau)\right\|_{L^{\infty}} d \tau}\left\|\rho_{0}\right\|_{H^{s-1}} \\
& \leq e^{2 \int_{0}^{t}\left\|\partial_{x} u(\tau)\right\|_{L^{\infty}} d \tau}\left\|\rho_{0}\right\|_{H^{s-1}} .
\end{aligned}
$$

Using similar arguments as in the proof of Lemma 18, one completes the proof of Lemma 19. 
4.1. The Proof of Theorem 2. We present the proof of Theorem 2 by inductive arguments with respect to the index $s(s>3 / 2)$.

Step 1. For $s \in(3 / 2,2)$, using Lemma 11 and the second equation in (6), one obtains

$$
\begin{aligned}
\|\rho(t)\|_{H^{s-1}} \leq & \left\|\rho_{0}\right\|_{H^{s-1}}+C \int_{0}^{t}\left\|u_{x} \rho\right\|_{H^{s-1}} d \tau \\
& +C \int_{0}^{t}\|\rho\|_{H^{s-1}}\left(\|u\|_{L^{\infty}}+\left\|u_{x}\right\|_{L^{\infty}}+\lambda\right) d \tau .
\end{aligned}
$$

From (17), we have

$$
\left\|\partial_{x} u \rho\right\|_{H^{s-1}} \leq C\left(\left\|\partial_{x} u\right\|_{H^{s-1}}\|\rho\|_{L^{\infty}}+\left\|\partial_{x} u\right\|_{L^{\infty}}\|\rho\|_{H^{s-1}}\right) .
$$

Thus

$$
\begin{aligned}
\|\rho(t)\|_{H^{s-1}} \leq & \left\|\rho_{0}\right\|_{H^{s-1}}+C \int_{0}^{t}\left\|\partial_{x} u\right\|_{H^{s-1}}\|\rho\|_{L^{\infty}} d \tau \\
& +C \int_{0}^{t}\|\rho\|_{H^{s-1}}\left(\|u\|_{L^{\infty}}+\left\|\partial_{x} u\right\|_{L^{\infty}}+\lambda\right) d \tau .
\end{aligned}
$$

On the other hand, using (3) of Lemma 9 and the first equation in (6) derives

$$
\begin{aligned}
\|u(t)\|_{H^{s}} \leq & \left\|u_{0}\right\|_{H^{s}}+C \int_{0}^{t}\left\|P(D)\left(\frac{3}{2} u^{2}+\frac{c}{2} \rho^{2}\right)-\lambda_{1} u\right\|_{H^{s}} d \tau \\
& +C \int_{0}^{t}\|u\|_{H^{s}}\left\|\partial_{x} u\right\|_{L^{\infty}} d \tau .
\end{aligned}
$$

Applying (7) of Proposition 8 yields

$$
\begin{aligned}
\| P(D) & \left(\frac{3}{2} u^{2}+\frac{c}{2} \rho^{2}\right)-\lambda_{1} u \|_{H^{s}} \\
\leq C & \left\|\frac{3}{2} u^{2}+\frac{c}{2} \rho^{2}\right\|_{H^{s-1}}+C \lambda_{1}\|u\|_{H^{s}} \\
\leq & C\left(\|u\|_{H^{s-1}}\|u\|_{L^{\infty}}+\|\rho\|_{H^{s-1}}\|\rho\|_{L^{\infty}}+\|u\|_{H^{s}}\right) .
\end{aligned}
$$

Hence

$$
\begin{aligned}
\|u(t)\|_{H^{s}} \leq & \left\|u_{0}\right\|_{H^{s}}+C \int_{0}^{t}\|u\|_{H^{s}}\left(\|u\|_{L^{\infty}}+\left\|\partial_{x} u\right\|_{L^{\infty}}+1\right) d \tau \\
& +C \int_{0}^{t}\|\rho\|_{H^{s-1}}\|\rho\|_{L^{\infty}} d \tau .
\end{aligned}
$$

Combining (84) and (87), one deduces

$$
\begin{aligned}
\|u(t)\|_{H^{s}} & +\|\rho(t)\|_{H^{s-1}} \\
\leq & \left\|u_{0}\right\|_{H^{s}}+\left\|\rho_{0}\right\|_{H^{s-1}} \\
& +C \int_{0}^{t}\left(\|u(t)\|_{H^{s}}+\|\rho(t)\|_{H^{s-1}}\right) \\
& \quad \times\left(\|u\|_{L^{\infty}}+\left\|\partial_{x} u\right\|_{L^{\infty}}+\|\rho\|_{L^{\infty}}+1\right) d \tau .
\end{aligned}
$$

Applying Gronwall's inequality yields

$$
\begin{aligned}
& \|u(t)\|_{H^{s}}+\|\rho(t)\|_{H^{s-1}} \\
& \quad \leq\left(\left\|u_{0}\right\|_{H^{s}}+\left\|\rho_{0}\right\|_{H^{s-1}}\right) e^{C \int_{0}^{t}\left(\|u\|_{L^{\infty}}+\left\|\partial_{x} u\right\|_{L^{\infty}}+\|\rho\|_{\left.L^{\infty}+1\right) d \tau}\right.} .
\end{aligned}
$$

Therefore, if $T<\infty$ satisfies $\int_{0}^{T}\left\|\partial_{x} u(\tau)\right\|_{L^{\infty}} d \tau<\infty$, from (89), Lemma 19, and the fact that $\|\rho(t, \cdot)\|_{L^{\infty}} \leq$ $e^{2 \int_{0}^{t}\left\|\partial_{x} u(\tau)\right\|_{L} \infty d \tau}\left\|\rho_{0}\right\|_{H^{s-1}}$, we have

$$
\begin{aligned}
& \|u(t)\|_{H^{s}}+\|\rho(t)\|_{H^{s-1}} \\
& \leq\left(\left\|u_{0}\right\|_{H^{s}}+\left\|\rho_{0}\right\|_{H^{s-1}}\right)
\end{aligned}
$$

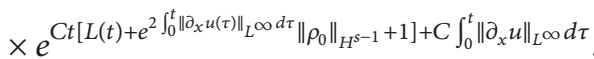

Thus

$$
\lim \sup _{t \rightarrow T^{-}}\left(\|u(t)\|_{H^{s}}+\|\rho(t)\|_{H^{s-1}}\right)<\infty,
$$

which contradicts the assumption that $T<\infty$ is the maximal existence time. This completes the proof for $s \in(3 / 2,2)$.

Step 2. For $s \in[2,5 / 2$ ), applying (1) of Lemma 9 to the second equation in (6) derives

$$
\begin{aligned}
\|\rho(t)\|_{H^{s-1}} \leq & \left\|\rho_{0}\right\|_{H^{s-1}}+C \int_{0}^{t}\left\|\partial_{x} u \rho\right\|_{H^{s-1}} d \tau \\
& +C \int_{0}^{t}\|\rho\|_{H^{s-1}}\left(\left\|\partial_{x} u\right\|_{H^{1 / 2} \cap L^{\infty}}+\lambda\right) d \tau .
\end{aligned}
$$

Thus

$$
\begin{aligned}
\|\rho(t)\|_{H^{s-1}} \leq & \left\|\rho_{0}\right\|_{H^{s-1}}+C \int_{0}^{t}\left\|u_{x}\right\|_{H^{s-1}}\|\rho\|_{L^{\infty}} d \tau \\
& +C \int_{0}^{t}\|\rho\|_{H^{s-1}}\left(\left\|u_{x}\right\|_{H^{1 / 2} \cap L^{\infty}}+\lambda\right) d \tau,
\end{aligned}
$$

which together with (87) and the fact that $H^{(1 / 2)+}(\mathbb{S}) \hookrightarrow$ $H^{1 / 2}(\mathbb{S}) \cap L^{\infty}(\mathbb{S})$, makes one deduce

$$
\begin{aligned}
& \|u(t)\|_{H^{s}}+\|\rho(t)\|_{H^{s-1}} \\
& \leq\left\|u_{0}\right\|_{H^{s}}+\left\|\rho_{0}\right\|_{H^{s-1}} \\
& \quad+C \int_{0}^{t}\left(\|u(t)\|_{H^{s}}+\|\rho(t)\|_{H^{s-1}}\right)\left(\|u\|_{H^{(3 / 2)+}}+\|\rho\|_{L^{\infty}}+1\right) d \tau .
\end{aligned}
$$

It follows from Gronwall's inequality that

$$
\begin{aligned}
& \|u(t)\|_{H^{s}}+\|\rho(t)\|_{H^{s-1}} \\
& \quad \leq\left(\left\|u_{0}\right\|_{H^{s}}+\left\|\rho_{0}\right\|_{H^{s-1}}\right) e^{C \int_{0}^{t}\left(\|u\|_{H^{(3 / 2)+}+}\|\rho\|_{L^{\infty}}+1\right) d \tau} .
\end{aligned}
$$

Therefore, if $T<\infty$ satisfies $\int_{0}^{T}\left\|\partial_{x} u(\tau)\right\|_{L^{\infty}} d \tau<\infty$, from (95) and $\|\rho(t, \cdot)\|_{L^{\infty}} \leq e^{2 \int_{0}^{t}\left\|\partial_{x} u(\tau)\right\|_{L^{\infty}} d \tau}\left\|\rho_{0}\right\|_{H^{s-1}}$, we obtain

$$
\lim \sup _{t \rightarrow T^{-}}\left(\|u(t)\|_{H^{s}}+\|\rho(t)\|_{H^{s-1}}\right)<\infty,
$$


which contradicts with the assumption that $T<\infty$ is the maximal existence time. This completes the proof for $s \in$ $[2,5 / 2)$.

Step 3. For $s \in(2,3)$, differentiating the second equation in (6) with respect to $x$, we obtain

$$
\partial_{t} \rho_{x}+u \partial_{x} \rho_{x}+3 u_{x} \rho_{x}+2 u_{x x} \rho+\lambda \rho_{x}=0 .
$$

Using Lemma 11 derives

$$
\begin{aligned}
\| \partial_{x} \rho(t) & \|_{H^{s-2}} \\
\leq & \left\|\partial_{x} \rho_{0}\right\|_{H^{s-2}}+C \int_{0}^{t}\left\|3 u_{x} \rho_{x}+2 u_{x x} \rho+\lambda \rho_{x}\right\|_{H^{s-2}} d \tau \\
& +C \int_{0}^{t}\left\|\partial_{x} \rho\right\|_{H^{s-2}}\left(\|u\|_{L^{\infty}}+\left\|\partial_{x} u\right\|_{L^{\infty}}\right) d \tau .
\end{aligned}
$$

Thanks to (6) of Proposition 8, one obtains

$$
\begin{gathered}
\left\|u_{x} \rho_{x}\right\|_{H^{s-2}} \leq C\left(\left\|\partial_{x} u\right\|_{H^{s-1}}\|\rho\|_{L^{\infty}}+\left\|\partial_{x} u\right\|_{L^{\infty}}\left\|\partial_{x} \rho\right\|_{H^{s-2}}\right), \\
\left\|\rho u_{x x}\right\|_{H^{s-2}} \leq C\left(\|\rho\|_{H^{s-1}}\left\|\partial_{x} u\right\|_{L^{\infty}}+\|\rho\|_{L^{\infty}}\left\|u_{x x}\right\|_{H^{s-2}}\right), \\
\left\|\lambda \rho_{x}\right\|_{H^{s-2}} \leq C \lambda\|\rho\|_{H^{s-1}} .
\end{gathered}
$$

Thus

$$
\begin{aligned}
& \left\|\partial_{x} \rho(t)\right\|_{H^{s-2}} \\
& \leq\left\|\partial_{x} \rho_{0}\right\|_{H^{s-2}} \\
& \quad+C \int_{0}^{t}\left(\|u\|_{H^{s}}+\|\rho\|_{H^{s-1}}\right) \\
& \quad \quad \times\left(\|u\|_{L^{\infty}}+\left\|\partial_{x} u\right\|_{L^{\infty}}+\|\rho\|_{L^{\infty}}+1\right) d \tau,
\end{aligned}
$$

which together with (87) and (84) with $s-2$ instead of $s-1$ derives

$$
\begin{aligned}
& \|u(t)\|_{H^{s}}+\|\rho(t)\|_{H^{s-1}} \\
& \leq\left\|u_{0}\right\|_{H^{s}}+\left\|\rho_{0}\right\|_{H^{s-1}} \\
& +C \int_{0}^{t}\left(\|u\|_{H^{s}}+\|\rho\|_{H^{s-1}}\right) \\
& \quad \quad \times\left(\|u\|_{L^{\infty}}+\left\|\partial_{x} u\right\|_{L^{\infty}}+\|\rho\|_{L^{\infty}}+1\right) d \tau .
\end{aligned}
$$

Similar to the arguments in Step 1, one completes the proof for $s \in(2,3)$.

Step 4. For $s=k_{1} \in \mathbb{N}$ and $k_{1} \geq 3$, differentiating the second equation in (6) $k_{1}-2$ times with respect to $x$ derives

$$
\begin{gathered}
\left(\partial_{t}+u \partial_{x}\right) \partial_{x}^{k_{1}-2} \rho+\sum_{l_{1}+l_{2}=k_{1}-3, l_{1}, l_{2} \geq 0} C_{l_{1}, l_{2}} \partial_{x}^{l_{1}+1} u \partial_{x}^{l_{2}+1} \rho \\
+2 \rho \partial_{x}^{k_{1}-1} u+\lambda \partial_{x}^{k_{1}-2} \rho=0 .
\end{gathered}
$$

From Lemma 9, we have

$$
\begin{gathered}
\left\|\partial_{x}^{k_{1}-2} \rho(t)\right\|_{H^{1}} \\
\leq\left\|\partial_{x}^{k_{1}-2} \rho_{0}\right\|_{H^{1}}+C \int_{0}^{t}\left\|\partial_{x}^{k_{1}-2} \rho\right\|_{H^{1}}\left\|\partial_{x} u\right\|_{H^{1 / 2} \cap L^{\infty}} d \tau \\
+C \int_{0}^{t} \| \sum_{l_{1}+l_{2}=k_{1}-3, l_{1}, l_{2} \geq 0} C_{l_{1}, l_{2}} \partial_{x}^{l_{1}+1} u \partial_{x}^{l_{1}+1} \rho \\
+2 \rho \partial_{x}^{k_{1}-1} u+\lambda \partial_{x}^{k_{1}-2} \rho \|_{H^{1}} d \tau .
\end{gathered}
$$

Using the algebraic properties of $H^{1}(\mathbb{S})$ derives

$$
\begin{gathered}
\left\|\rho \partial_{x}^{k_{1}-1} u\right\|_{H^{1}} \leq C\|\rho\|_{H^{1}}\left\|\partial_{x}^{k_{1}-1}\right\|_{H^{1}} \leq C\|\rho\|_{H^{1}}\|u\|_{H^{s}}, \\
\left\|\sum_{l_{1}+l_{2}=k_{1}-3, l_{1}, l_{2} \geq 0} C_{l_{1}, l_{2}} \partial_{x}^{l_{1}+1} u \partial_{x}^{l_{2}+1} \rho\right\|_{H^{1}} \leq C\|u\|_{H^{s-1}}\|\rho\|_{H^{s-1}}, \\
\left\|\partial_{x}^{k_{1}-2} \rho\right\|_{H^{1}} \leq C\|\rho\|_{H^{s-1}} .
\end{gathered}
$$

Thus

$$
\begin{aligned}
& \left\|\partial_{x}^{k_{1}-2} \rho(t)\right\|_{H^{1}} \\
& \leq\left\|\partial_{x}^{k_{1}-2} \rho_{0}\right\|_{H^{1}} \\
& \quad+C \int_{0}^{t}\left(\|u\|_{H^{s}}+\|\rho\|_{H^{s-1}}\right) \times\left(\|u\|_{H^{s-1}}+\|\rho\|_{H^{1}}+1\right) d \tau,
\end{aligned}
$$

which together with (87), (84) with $s-1$ instead of 1 derives

$$
\begin{aligned}
\|u(t)\|_{H^{s}} & +\|\rho(t)\|_{H^{s-1}} \\
\leq & C\left(\left\|u_{0}\right\|_{H^{s}}+\left\|\rho_{0}\right\|_{H^{s-1}}\right) \\
& +C \int_{0}^{t}\left(\|u(t)\|_{H^{s}}+\|\rho(t)\|_{H^{s-1}}\right) \\
& \quad \times\left(\|u(t)\|_{H^{s-1}}+\|\rho(t)\|_{H^{1}}+1\right) d \tau .
\end{aligned}
$$

Using Gronwall's inequality, we obtain

$$
\begin{aligned}
& \|u(t)\|_{H^{s}}+\|\rho(t)\|_{H^{s-1}} \\
& \quad \leq C\left(\left\|u_{0}\right\|_{H^{s}}+\left\|\rho_{0}\right\|_{H^{s-1}}\right) e^{C \int_{0}^{t}\left(\|u\|_{H^{s-1}}+\|\rho\|_{H^{1}}+1\right) d \tau} .
\end{aligned}
$$

If $T<\infty$ satisfies $\int_{0}^{T}\left\|\partial_{x} u(\tau)\right\|_{L^{\infty}} d \tau<\infty$, using the uniqueness of solutions in Theorem 1, one obtains that $\|u\|_{H^{s-1}}+$ $\|\rho\|_{H^{1}}$ is uniformly bounded. Then

$$
\lim \sup _{t \rightarrow T^{-}}\left(\|u(t)\|_{H^{s}}+\|\rho(t)\|_{H^{s-1}}\right)<\infty,
$$


which contradicts the assumption that the maximal existence time $T<\infty$. This completes the proof for $s=k_{1} \in \mathbb{N}$ and $k_{1} \geq 3$.

Step 5. For $s \in\left(k_{1}, k_{1}+1\right), k_{1} \in \mathbb{N}$ and $k_{1} \geq 3$, differentiating the second equation in system (6) $k_{1}-1$ times with respect to $x$, we obtain

$$
\begin{aligned}
\left(\partial_{t}+u \partial_{x}\right) \partial_{x}^{k_{1}-1} \rho+\sum_{l_{1}+l_{2}=k_{1}-2, l_{1}, l_{2} \geq 0} C_{l_{1}, l_{2}} \partial_{x}^{l_{1}+1} u \partial_{x}^{l_{2}+1} \rho \\
+2 \rho \partial_{x}^{k_{1}} u+\lambda \partial_{x}^{k_{1}-1} \rho=0 .
\end{aligned}
$$

Using Lemma 11 with $s-k_{1} \in(0,1)$, one derives

$$
\begin{aligned}
& \left\|\partial_{x}^{k_{1}-1} \rho(t)\right\|_{H^{s-k_{1}}} \\
& \leq\left\|\partial_{x}^{k_{1}-1} \rho_{0}\right\|_{H^{s-k_{1}}} \\
& +C \int_{0}^{t}\left\|\partial_{x}^{k_{1}-1} \rho\right\|_{H^{s-k_{1}}}\left(\left\|\partial_{x} u\right\|_{L^{\infty}}+\|u\|_{L^{\infty}}\right) d \tau \\
& +C \int_{0}^{t} \| \sum_{l_{1}+l_{2}=k_{1}-2, l_{1}, l_{2} \geq 0} C_{l_{1}, l_{2}} \partial_{x}^{l_{1}+1} u \partial_{x}^{l_{2}+1} \rho \\
& +2 \rho \partial_{x}^{k_{1}} u+\lambda \partial_{x}^{k_{1}-1} \rho \|_{H^{s-k_{1}}} d \tau .
\end{aligned}
$$

For sufficiently small $\varepsilon>0$, using (19) and the fact that $H^{1 / 2+\varepsilon}(\mathbb{S}) \hookrightarrow L^{\infty}(\mathbb{S})$, one has

$$
\begin{gathered}
\left\|\rho \partial_{x}^{k_{1}} u\right\|_{H^{s-k_{1}}} \\
\leq C\left(\left\|\partial_{x}^{k_{1}} u\right\|_{H^{s-k_{1}}}\|\rho\|_{L^{\infty}}+\left\|\partial_{x}^{k_{1}-1} u\right\|_{L^{\infty}}\|\rho\|_{H^{s-k_{1}+1}}\right) \\
\leq C\left(\|u\|_{H^{s}}\|\rho\|_{L^{\infty}}+\|u\|_{H^{k_{1}-1 / 2+\varepsilon}}\|\rho\|_{H^{s-k_{1}+1}}\right), \\
\sum_{l_{l_{1}+l_{2}=k_{1}-2, l_{1}, l_{2} \geq 0} C_{l_{1}, l_{2}} \partial_{x}^{l_{1}+1} u \partial_{x}^{l_{2}+1} \rho \|_{H^{s-k_{1}}}} \sum_{l_{1}+l_{2}=k_{1}-2, l_{1}, l_{2} \geq 0} C_{l_{1}, l_{2}}\left(\left\|\partial_{x}^{l_{1}+1} u\right\|_{L^{\infty}}\left\|\partial_{x}^{l_{2}+1} \rho\right\|_{H^{s-k_{1}}}\right. \\
\leq C\left(\|u\|_{H^{k_{1}-1 / 2+\varepsilon}}\|\rho\|_{H^{s-1}}+\|u\|_{H^{s}}\|\rho\|_{H^{k_{1}-3 / 2+\varepsilon}}\right), \\
\left\|\lambda \partial_{x}^{k_{1}-1} \rho\right\|_{H^{s-k_{1}}} \leq C\|\rho\|_{H^{s-1}} .
\end{gathered}
$$

Making use of (110) and (111) yields

$$
\begin{aligned}
& \left\|\partial_{x}^{k_{1}-1} \rho(t)\right\|_{H^{s-k_{1}}} \\
& \leq\left\|\partial_{x}^{k_{1}-1} \rho_{0}\right\|_{H^{s-k_{1}}} \\
& \quad+C \int_{0}^{t}\left(\|u\|_{H^{s}}+\|\rho\|_{H^{s-1}}\right)\left(\|u\|_{H^{k_{1}-1 / 2+\varepsilon}}+\|\rho\|_{H^{k_{1}-3 / 2+\varepsilon}}+1\right) d \tau,
\end{aligned}
$$

which together with (87) and (84) with $s-k_{1} \in(0,1)$ instead of $s-1$ derives

$$
\begin{aligned}
& \|u(t)\|_{H^{s}}+\|\rho(t)\|_{H^{s-1}} \\
& \leq C\left(\left\|u_{0}\right\|_{H^{s}}+\left\|\rho_{0}\right\|_{H^{s-1}}\right) \\
& \quad+C \int_{0}^{t}\left(\|u\|_{H^{s}}+\|\rho\|_{H^{s-1}}\right)\left(\|u\|_{H^{k_{1}-1 / 2+\varepsilon}}+\|\rho\|_{H^{k_{1}-3 / 2+\varepsilon}}+1\right) d \tau .
\end{aligned}
$$

Thanks to Gronwall's inequality, one has

$$
\begin{aligned}
& \|u(t)\|_{H^{s}}+\|\rho(t)\|_{H^{s-1}} \\
& \quad \leq C\left(\left\|u_{0}\right\|_{H^{s}}+\left\|\rho_{0}\right\|_{H^{s-1}}\right) e^{C \int_{0}^{t}\left(\|u\|_{H^{k_{1}-1 / 2+\varepsilon}}+\|\rho\|_{H^{k_{1}-3 / 2+\varepsilon}}+1\right) d \tau} .
\end{aligned}
$$

Using the uniqueness of solutions in Theorem 1, we obtain that

$$
\|u\|_{H^{k_{1}-1 / 2+\varepsilon}}+\|\rho\|_{H^{k_{1}-3 / 2+\varepsilon}}
$$

is uniformly bounded by the induction assumption. Then

$$
\lim \sup _{t \rightarrow T^{-}}\left(\|u(t)\|_{H^{s}}+\|\rho(t)\|_{H^{s-1}}\right)<\infty,
$$

which leads to a contradiction.

Thus from Step 1 to Step 5, one completes the proof of Theorem 2.

4.2. The Proof of Theorem 3. Using simple density arguments, here we only need to prove the theorem for $s=3$. Assume that there exists $T>0$ and $M>0$ such that

$$
u_{x}(t, x) \geq-M, \quad \forall(t, x) \in[0, T] \times \mathbb{S} .
$$

Applying Lemma 17 yields

$$
\|\rho(t, \cdot)\|_{L^{\infty}},\|\rho(t, \cdot)\|_{L^{2}} \leq e^{2 M t}\left\|\rho_{0}\right\|_{H^{s-1}}, \quad \forall t \in[0, T] .
$$

Differentiating the first equation in (6) with respect to $x$ and using $\partial_{x}^{2} g * f=g * f-f$ yield

$$
\begin{aligned}
u_{t x}= & -u_{x}^{2}-u u_{x x}-g *\left(\frac{3}{2} u^{2}+\frac{c}{2} \rho^{2}\right)-\lambda_{1} u_{x} \\
& +\left(\frac{3}{2} u^{2}+\frac{c}{2} \rho^{2}\right) .
\end{aligned}
$$


Noting

$$
\begin{aligned}
\frac{d u_{x}(t, q(t, x))}{d t} & =u_{x t}(t, q(t, x))+u_{x x}(t, q(t, x)) q_{t}(t, x) \\
& =\left(u_{t x}+u u_{x x}\right)(t, q(t, x))
\end{aligned}
$$

and combining (119), (120), and $u_{x}^{2} \geq 0, g * u^{2} \geq 0$, $\left\|g * \rho^{2}\right\|_{L^{\infty}} \leq\|g\|_{L^{1}}\|\rho\|_{L^{\infty}}^{2} \leq c_{0}\|\rho\|_{L^{\infty}}^{2},(62),(66)$, one deduces

$$
\begin{aligned}
& \frac{d u_{x}(t, q(t, x))}{d t} \\
& =\left[-u_{x}^{2}-g *\left(\frac{3}{2} u^{2}+\frac{c}{2} \rho^{2}\right)-\lambda_{1} u_{x}+\left(\frac{3}{2} u^{2}+\frac{c}{2} \rho^{2}\right)\right](t, q) \\
& \leq \frac{|c|}{2} c_{0}\|\rho\|_{L^{\infty}}^{2}+\lambda_{1} M+\frac{3}{2}\|u\|_{L^{\infty}}^{2}+\frac{|c|}{2}\|\rho\|_{L^{\infty}}^{2} .
\end{aligned}
$$

Using Lemmas 17 and 18, one deduces that there exists $C_{4}>0$ such that

$$
\frac{d u_{x}(t, q(t, x))}{d t} \leq C_{4}
$$

For $t \in(0, T)$, integrating the above inequality with respect to $t$ on interval $(0, t)$, we have

$$
u_{x}(t, q(t, x)) \leq u_{0 x}(x)+C_{4} t .
$$

Thus

$$
\begin{array}{r}
\sup _{x \in \mathbb{S}} u_{x}(t, x) \leq\left\|\partial_{x} u_{0}\right\|_{L^{\infty}}+C_{4} t \leq\left\|u_{0}\right\|_{H^{s}}+C_{4} T, \\
\forall t \in[0, T],
\end{array}
$$

which together with (117) and $T<\infty$ derives

$$
\int_{0}^{T}\left\|\partial_{x} u(\tau)\right\|_{L^{\infty}} d \tau<\infty .
$$

This contradicts with the results in Theorem 2 .

On the other hand, applying Sobolev's embedding theorem, one deduces

$$
\lim \inf _{t \rightarrow T^{-}} \inf _{x \in \mathbb{S}} u_{x}(t, x)=-\infty,
$$

and then the solution $(u, \rho)$ blows up in finite time. This completes the proof.

Remark 20. Theorem 3 implies that the blow-up phenomenon of solution $(u, \rho)$ to system (6) only depends on the slope of the first component $u$. In other words, the first component $u$ blows up before the second component $\rho$ in finite time.

4.3. The Proof of Theorem 4. We use Lemmas 17 and 18 to prove Theorem 4. For simplicity, we assume $s=3$ here. Noting the assumption $u_{0}$ is odd, $\rho_{0}$ is even, and the structure of system (6), one deduces that $u(t, x)$ is odd and $\rho(t, x)$ is even with respect to $x$ for all $t \in(0, T)$. Thus $u(t, 0)=0$ and $\rho_{x}(t, 0)=0$. Thanks to the second equation in system (6) at the point $x=0$, we have

$$
\begin{gathered}
\frac{d}{d t} \rho(t, 0)=\left[-2 u_{x}(t, 0)-\lambda\right] \rho(t, 0), \quad t>0, x \in \mathbb{S}, \\
\rho(0,0)=0, \quad x \in \mathbb{S},
\end{gathered}
$$

which derives $\rho(t, 0)=0$.

Differentiating the first equation in system (6) with respect to variable $x$ yields

$$
u_{x t}=-u_{x}^{2}-u u_{x x}-g *\left(\frac{3}{2} u^{2}+\frac{c}{2} \rho^{2}\right)+\frac{3}{2} u^{2}+\frac{c}{2} \rho^{2}-\lambda_{1} u_{x} .
$$

Noting the assumption $c \geq 0$ in Theorem 4 , one obtains $g *$ $\left((3 / 2) u^{2}+(c / 2) \rho^{2}\right) \geq 0$. Setting $M(t)=u_{x}(t, 0)+\lambda_{1} / 2$ and combining with (128) yield

$$
\frac{d M(t)}{d t} \leq-M^{2}(t)+\frac{\lambda_{1}^{2}}{4}
$$

By the assumption $M(0)=u_{0 x}(0)+\lambda_{1} / 2<-\lambda_{1} / 2$, we have $M^{2}(0)>\lambda_{1}^{2} / 4$. We claim that $M(t)<-\lambda_{1} / 2$ is true for all $t \in[0, T]$. In fact, if the claim is not true for all $t \in[0, T]$, then from the continuity of $M(t)$, we deduce that there exists $t_{0} \in$ $(0, T)$ such that $M^{2}(t)>\lambda_{1}^{2} / 4$ for $t \in\left[0, t_{0}\right]$, and $M^{2}\left(t_{0}\right)=$ $\lambda_{1}^{2} / 4$. Combining this with (129) derives $d M(t) / d t<0$ a.e. on $\left[0, t_{0}\right]$. Since $M(t)$ is absolutely continuous on $\left[0, t_{0}\right]$, one gets the contradiction $M\left(t_{0}\right)<M(0)=u_{0 x}\left(x_{0}\right)+\lambda_{1} / 2<-\lambda_{1} / 2$. This completes the proof of the claim.

Thus we obtain that $M(t)$ is strictly decreasing on $[0, T]$. Let $\delta \in(0,1)$ such that $-\sqrt{\delta} M(0)=\lambda_{1} / 2$. From (129), we have

$$
\begin{aligned}
\frac{d M(t)}{d t} & \leq-M^{2}(t)+\delta M^{2}(0) \\
& \leq-(1-\delta) M^{2}(t) \quad \text { a.e. on }[0, T] .
\end{aligned}
$$

Since $M(t)$ is locally Lipschitz on $[0, T]$ and strictly negative, thus $1 / M(t)$ is also locally Lipschitz on $[0, T]$. It follows that

$$
\frac{d}{d t}\left[\frac{1}{M(t)}\right]=-\frac{1}{M^{2}(t)} \frac{d M(t)}{d t} \geq 1-\delta \quad \text { a.e. on }[0, T] \text {. }
$$

Integrating (131) with respect to $t$ over $(0, t)$ yields

$$
-\frac{1}{M(t)}+\frac{1}{M(0)} \leq-(1-\delta) t \quad \text { a.e. on }[0, T] .
$$

Since $M(t)<0$ on $[0, T]$, one obtains that the maximal existence time $T \leq-1 /(1-\delta) M(0)<\infty$. Moreover, using the assumption $M(0)=u_{0 x}(0)+\lambda_{1} / 2<0$ derives

$$
\begin{array}{r}
u_{x}(t, 0) \leq \frac{u_{0 x}(0)+\lambda_{1} / 2}{1+t(1-\delta)\left(u_{0 x}(0)+\lambda_{1} / 2\right)}-\frac{\lambda_{1}}{2} \longrightarrow-\infty, \\
t \longrightarrow-\frac{1}{(1-\delta)\left(u_{0 x}(0)+\lambda_{1} / 2\right)},
\end{array}
$$

which completes the first part proof of Theorem 4. 
On the other hand, differentiating the second equation in (6) with respect to $x$ yields

$$
\frac{d \rho_{x}(t, q(t, x))}{d t}=\left(-3 u_{x} \rho_{x}-2 u_{x x} \rho-\lambda \rho_{x}\right)(t, q(t, x)) \text {. }
$$

Taking $x=x_{1}(t)$ and noting $q\left(t, x_{1}(t)\right)=\xi(t)$, together with the definition of $m(t)$ in Lemma 15, one deduces $u_{x x}(t, \xi(t))=$ 0 a.e. $t \in[0, T]$. Thus

$$
\frac{d \rho_{x}(t, \xi(t))}{d t}=\left[-3 u_{x}(t, \xi(t))-\lambda\right] \rho_{x}(t, \xi(t)) .
$$

Using the assumption $u_{0 x}\left(x_{0}\right)=\inf _{x \in \mathbb{S}} u_{0 x}(x)$ in Theorem 4, (57) and letting $\xi(0)=x_{0}$ yield $\rho_{0 x}(\xi(0))=\rho_{0 x}\left(x_{0}\right)$. From (135), one deduces

$$
\begin{aligned}
\rho_{x}(t, \xi(t)) & =\rho_{0 x}\left(x_{0}\right) e^{\int_{0}^{t}\left(-3 u_{x}(s, \xi(s))-\lambda\right) d s} \\
& =\rho_{0 x}\left(x_{0}\right) e^{\int_{0}^{t}\left(-3 \inf _{x \in \mathcal{S}} u_{x}(s, x)-\lambda\right) d s} .
\end{aligned}
$$

Thanks to (133), for all $t \in[0, T]$, we have

$$
\begin{aligned}
& e^{\int_{0}^{t}\left(-3 \inf _{x \in \mathbb{S}} u_{x}(s, x)-\lambda\right) d s} \\
& \quad \geq e^{\int_{0}^{t}\left[-3\left(\left(u_{0 x}(0)+\lambda_{1} / 2\right) /\left(1+s(1-\delta)\left(u_{0 x}(0)+\lambda_{1} / 2\right)\right)-\lambda_{1} / 2\right)-\lambda\right] d s} \\
& \quad=e^{\left(3 \lambda_{1} / 2-\lambda\right) t-(3 /(1-\delta)) \ln \left[1+(1-\delta)\left(u_{0 x}(0)+\lambda_{1} / 2\right) t\right]} .
\end{aligned}
$$

Note $e^{\left(3 \lambda_{1} / 2-\lambda\right) t-(3 /(1-\delta)) \ln \left[1+(1-\delta)\left(u_{0 x}(0)+\lambda_{1} / 2\right) t\right]} \rightarrow \infty$ as $t \rightarrow$ $-1 /(1-\delta)\left(u_{0 x}(0)+\lambda_{1} / 2\right)$. Thus, if $\rho_{0 x}\left(x_{0}\right)>0$, then from (136), for $T_{1} \in\left(0,-1 /(1-\delta)\left(u_{0 x}(0)+\lambda_{1} / 2\right)\right]$, one has

$$
\sup _{x \in \mathbb{S}} \rho_{x}(t, x) \geq \rho_{x}(t, \xi(t)) \longrightarrow+\infty \quad \text { as } t \longrightarrow T_{1}^{-} .
$$

On the other hand, if $\rho_{0 x}\left(x_{0}\right)<0$, it follows from (136) that, for $T_{1} \in\left(0,-1 /(1-\delta)\left(u_{0 x}(0)+\lambda_{1} / 2\right)\right]$, one deduces

$$
\inf _{x \in \mathbb{S}} \rho_{x}(t, x) \leq \rho_{x}(t, \xi(t)) \longrightarrow-\infty \quad \text { as } t \longrightarrow T_{1}^{-} .
$$

This completes the proof of Theorem 4 .

4.4. The Proof of Theorem 5. Let $X(t, x)=(u(t, x), \rho(t, x))$ be the corresponding solution to system (1) with initial data $\left(u_{0}, \rho_{0}\right)$. Differentiating the first equation in (1) with respect to $x$, one has

$$
\begin{aligned}
u_{x t}= & -u_{x}^{2}-u u_{x x}-g *\left(\frac{3}{2} u^{2}+\frac{c}{2} \rho^{2}\right)+\frac{3}{2} u^{2}+\frac{c}{2} \rho^{2} \\
& -\lambda_{1} u_{x} .
\end{aligned}
$$

Differentiating the second equation in system (6) with respect to $x$ yields

$$
\rho_{x t}=-u \rho_{x x}-2 u_{x x} \rho-\left(3 u_{x}+\lambda\right) \rho_{x} .
$$

We obtain that $-X(t,-x)$ is also a solution to system (1) provided that $X(t, x)$ is a solution to system (1). Note the initial data $u_{0}$ and $\rho_{0}$ are odd; one derives $-X(0,-x)=$ $X(0, x)$. Using the uniqueness of solutions yields $-X(t,-x)=$ $X(t, x)$, and $X(t, x)$ is odd for all $t \in[0, T]$. Thus $u(t, 0)=$ $u_{x x}(t, 0)=\rho(t, 0)=0$ for all $t \in[0, T]$. From the above analysis and (140), we have

$$
\frac{d u_{x}(t, 0)}{d t} \leq-u_{x}^{2}(t, 0)-\lambda_{1} u_{x}(t, 0) .
$$

Similar to the proof of Theorem 4, we complete the first part proof of Theorem 5. From (141), one deduces

$$
\rho_{x t}(t, 0)=\left(-3 u_{x}(t, 0)-\lambda\right) \rho_{x}(t, 0), \quad t \in[0, T] .
$$

From (143), we get

$$
\rho_{x}(t, 0)=\rho_{0 x}(0) e^{\int_{0}^{t}\left(-3 u_{x}(\tau, 0)-\lambda\right) d \tau} .
$$

As before, we also obtain that $u_{x}(t, 0)$ is decreasing with $u_{0 x}(0)<-\lambda_{1}$. Thus $-u_{x}(t, 0)>-u_{0 x}(0)>0$, which combined with (144) completes the proof of Theorem 5.

\section{Conflict of Interests}

The authors declare that there is no conflict of interests regarding the publication of this paper.

\section{Acknowledgments}

This work is supported by National Natural Science Foundation of China (71003082) and Fundamental Research Funds for the Central Universities (SWJTU12CX061 and SWJTU09ZT36).

\section{References}

[1] A. Degasperis and M. Procesi, "Asymptotic integrability," in Symmetry and Perturbation Theory, pp. 23-37, World Scientific, Singapore, 1999.

[2] A. Degasperis, D. D. Kholm, and A. N. I. Khon, "A new integrable equation with peakon solutions," Theoretical and Mathematical Physics, vol. 133, no. 2, pp. 1463-1474, 2002.

[3] H. R. Dullin, G. A. Gottwald, and D. D. Holm, "Camassa-Holm, Korteweg-de Vries-5 and other asymptotically equivalent equations for shallow water waves," Fluid Dynamics Research, vol. 33, no. 1-2, pp. 73-79, 2003.

[4] J. Escher and B. Kolev, "The Degasperis-Procesi equation as a non-metric Euler equation," Mathematische Zeitschrift, vol. 269, no. 3-4, pp. 1137-1153, 2011.

[5] J. Escher and J. Seiler, "The periodic $b$-equation and Euler equations on the circle," Journal of Mathematical Physics, vol. 51, no. 5, Article ID 053101, 2010.

[6] J. Escher, Y. Liu, and Z. Yin, "Global weak solutions and blowup structure for the Degasperis-Procesi equation," Journal of Functional Analysis, vol. 241, no. 2, pp. 457-485, 2006.

[7] V. O. Vakhnenko and E. J. Parkes, "Periodic and solitary-wave solutions of the Degasperis-Procesi equation," Chaos, Solitons \& Fractals, vol. 20, no. 5, pp. 1059-1073, 2004.

[8] H. Lundmark and J. Szmigielski, "Multi-peakon solutions of the Degasperis-Procesi equation," Inverse Problems, vol. 19, no. 6, pp. 1241-1245, 2003. 
[9] H. Lundmark, "Formation and dynamics of shock waves in the Degasperis-Procesi equation," Journal of Nonlinear Science, vol. 17, no. 3, pp. 169-198, 2007.

[10] S. Lai and Y. Wu, "A model containing both the Camassa-Holm and Degasperis-Procesi equations," Journal of Mathematical Analysis and Applications, vol. 374, no. 2, pp. 458-469, 2011.

[11] L. Tian, Y. Chen, Y. Liu, and Y. Gao, "Low-regularity solutions of the periodic general Degasperis-Procesi equation," Nonlinear Analysis: Theory, Methods \& Applications, vol. 74, no. 8, pp. 2802-2812, 2011.

[12] X. Ai and G. Gui, "Global well-posedness for the Cauchy problem of the viscous Degasperis-Procesi equation," Journal of Mathematical Analysis and Applications, vol. 361, no. 2, pp. 457465, 2010.

[13] Y. Liu and Z. Yin, "Global existence and blow-up phenomena for the Degasperis-Procesi equation," Communications in Mathematical Physics, vol. 267, no. 3, pp. 801-820, 2006.

[14] Y. Matsuno, "Parametric representation for the multisoliton solution of the Camassa-Holm equation," Journal of the Physical Society of Japan, vol. 74, no. 7, pp. 1983-1987, 2005.

[15] Y. Matsuno, "The $N$-soliton solution of the Degasperis-Procesi equation," Inverse Problems, vol. 21, no. 6, pp. 2085-2101, 2005.

[16] Z. Lin and Y. Liu, "Stability of peakons for the DegasperisProcesi equation," Communications on Pure and Applied Mathematics, vol. 62, no. 1, pp. 125-146, 2009.

[17] Z. Yin, "On the Cauchy problem for an integrable equation with peakon solutions," Illinois Journal of Mathematics, vol. 47, no. 3 , pp. 649-666, 2003.

[18] Z. Yin, "Global weak solutions for a new periodic integrable equation with peakon solutions," Journal of Functional Analysis, vol. 212, no. 1, pp. 182-194, 2004.

[19] J. Escher, Y. Liu, and Z. Yin, "Shock waves and blow-up phenomena for the periodic Degasperis-Procesi equation," Indiana University Mathematics Journal, vol. 56, no. 1, pp. 87-117, 2007.

[20] Y. Guo, S. Lai, and Y. Wang, "Global weak solutions to the weakly dissipative Degasperis-Procesi equation," Nonlinear Analysis: Theory, Methods \& Applications, vol. 74, no. 15, pp. 4961-4973, 2011.

[21] S. Wu and Z. Yin, "Blow-up and decay of the solution of the weakly dissipative Degasperis-Procesi equation," SIAM Journal on Mathematical Analysis, vol. 40, no. 2, pp. 475-490, 2008.

[22] S. Wu, J. Escher, and Z. Yin, "Global existence and blowup phenomena for a weakly dissipative Degasperis-Procesi equation," Discrete and Continuous Dynamical Systems B, vol. 12, no. 3, pp. 633-645, 2009.

[23] Z. Guo, "Some properties of solutions to the weakly dissipative Degasperis-Procesi equation," Journal of Differential Equations, vol. 246, no. 11, pp. 4332-4344, 2009.

[24] Y. Fu and C. Qu, "Unique continuation and persistence properties of solutions of the 2-component Degasperis-Procesi equations," Acta Mathematica Scientia B, vol. 32, no. 2, pp. 652662, 2012.

[25] L. Jin and Z. Guo, "On a two-component Degasperis-Procesi shallow water system," Nonlinear Analysis: Real World Applications, vol. 11, no. 5, pp. 4164-4173, 2010.

[26] M. Yuen, "Self-similar blowup solutions to the 2-component Degasperis-Procesi shallow water system," Communications in Nonlinear Science and Numerical Simulation, vol. 16, no. 9, pp. 3463-3469, 2011.

[27] J. Zhou, L. Tian, and X. Fan, "Soliton, kink and antikink solutions of a 2-component of the Degasperis-Procesi equation,"
Nonlinear Analysis: Real World Applications, vol. 11, no. 4, pp. 2529-2536, 2010.

[28] K. Yan and Z. Yin, "On the Cauchy problem for a twocomponent Degasperis-Procesi system," Journal of Differential Equations, vol. 252, no. 3, pp. 2131-2159, 2012.

[29] Z. Popowicz, "A two-component generalization of the Degasperis-Procesi equation," Journal of Physics A: Mathematical and General, vol. 39, no. 44, pp. 13717-13726, 2006.

[30] J. Escher, O. Lechtenfeld, and Z. Yin, "Well-posedness and blowup phenomena for the 2-component Camassa-Holm equation," Discrete and Continuous Dynamical Systems A, vol. 19, no. 3, pp. 493-513, 2007.

[31] A. Constantin and R. I. Ivanov, "On an integrable twocomponent Camassa-Holm shallow water system," Physics Letters. A, vol. 372, no. 48, pp. 7129-7132, 2008.

[32] G. Gui and Y. Liu, "On the global existence and wave-breaking criteria for the two-component Camassa-Holm system," Journal of Functional Analysis, vol. 258, no. 12, pp. 4251-4278, 2010.

[33] G. Gui and Y. Liu, "On the Cauchy problem for the twocomponent Camassa-Holm system," Mathematische Zeitschrift, vol. 268, no. 1-2, pp. 45-66, 2011.

[34] M. Chen, S. Liu, and Y. Zhang, "A two-component generalization of the Camassa-Holm equation and its solutions," Letters in Mathematical Physics, vol. 75, no. 1, pp. 1-15, 2006.

[35] W. Chen, X. Deng, and J. Zhang, "Blow up and blow-up rate for the generalized 2-component Camassa-Holm equation," International Journal of Nonlinear Science, vol. 12, no. 3, pp. 313322, 2011.

[36] K. Yan and Z. Yin, "Analytic solutions of the Cauchy problem for two-component shallow water systems," Mathematische Zeitschrift, vol. 269, no. 3-4, pp. 1113-1127, 2011.

[37] C. Guan and Z. Yin, "Global existence and blow-up phenomena for an integrable two-component Camassa-Holm shallow water system," Journal of Differential Equations, vol. 248, no. 8, pp. 2003-2014, 2010.

[38] C. Guan and Z. Yin, "Global weak solutions for a twocomponent Camassa-Holm shallow water system," Journal of Functional Analysis, vol. 260, no. 4, pp. 1132-1154, 2011.

[39] W. Chen, L. Tian, X. Deng, and J. Zhang, "Wave breaking for a generalized weakly dissipative 2-component Camassa-Holm system," Journal of Mathematical Analysis and Applications, vol. 400, no. 2, pp. 406-417, 2013.

[40] Q. Hu, "Global existence and blow-up phenomena for a weakly dissipative periodic 2-component Camassa-Holm system," Journal of Mathematical Physics, vol. 52, no. 10, Article ID 103701, 2011.

[41] J. Lenells and M. Wunsch, "On the weakly dissipative CamassaHolm, Degasperis-Procesi, and Novikov equations," Journal of Differential Equations, vol. 255, no. 3, pp. 441-448, 2013.

[42] Y. Mi and C. Mu, "On the Cauchy problem for the modified Novikov equation with peakon solutions," Journal of Differential Equations, vol. 254, no. 3, pp. 961-982, 2013.

[43] J. Liu, "The Cauchy problem of a periodic 2-component $\mu$ Hunter-Saxton system in Besov spaces," Journal of Mathematical Analysis and Applications, vol. 399, no. 2, pp. 650-666, 2013.

[44] H. Bahouri, J. Chemin, and R. Danchin, "Fourier analysis and nonlinear partial differential equations," in Grundlehren der Mathematischen Wissenschaften, vol. 343, Springer, 2010.

[45] R. Danchin, "A few remarks on the Camassa-Holm equation," Differential and Integral Equations, vol. 14, no. 8, pp. 953-988, 2001. 
[46] R. Danchin, "Fourier analysis methods for PDEs, Lecture notes," 2005.

[47] W. Yan, Y. Li, and Y. Zhang, "The Cauchy problem for the integrable Novikov equation," Journal of Differential Equations, vol. 253, no. 1, pp. 298-318, 2012.

[48] W. Arendt and S. Bu, "Operator-valued Fourier multipliers on periodic Besov spaces and applications," Proceedings of the Edinburgh Mathematical Society II, vol. 47, no. 1, pp. 15-33, 2004.

[49] O. Glass, "Controllability and asymptotic stabilization of the Camassa-Holm equation," Journal of Differential Equations, vol. 245, no. 6, pp. 1584-1615, 2008.

[50] X. Wu and Z. Yin, "Well-posedness and blow-up phenomena for the generalized Degasperis-Procesi equation," Nonlinear Analysis: Theory, Methods \& Applications, vol. 73, no. 1, pp. 136146, 2010.

[51] A. Constantin and J. Escher, "Wave breaking for nonlinear nonlocal shallow water equations," Acta Mathematica, vol. 181, no. 2, pp. 229-243, 1998.

[52] A. Constantin, "Global existence of solutions and breaking waves for a shallow water equation, a geometric approach," Annales de l'Institut Fourier Universite de Grenoble, vol. 50, pp. 321-362, 2000. 


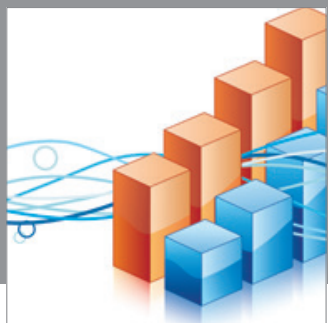

Advances in

Operations Research

mansans

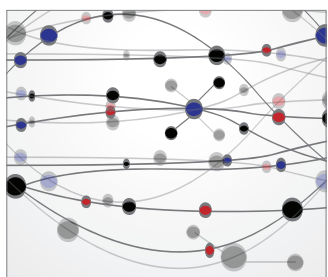

The Scientific World Journal
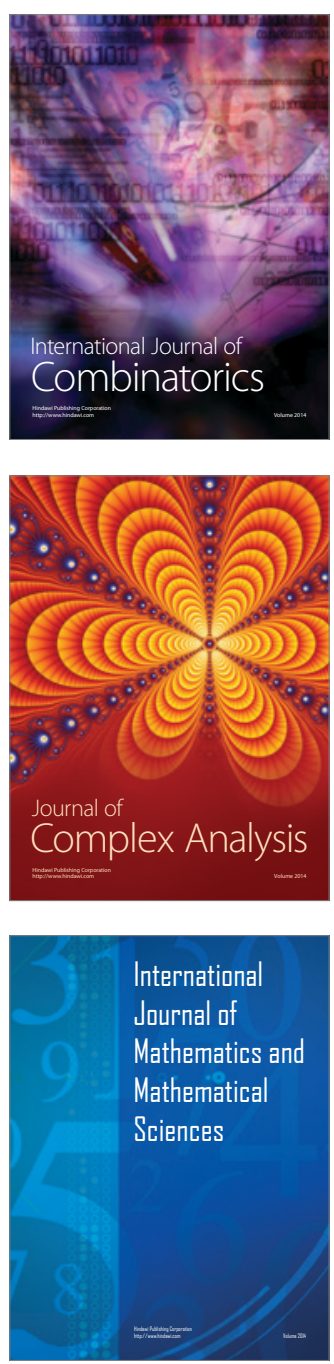
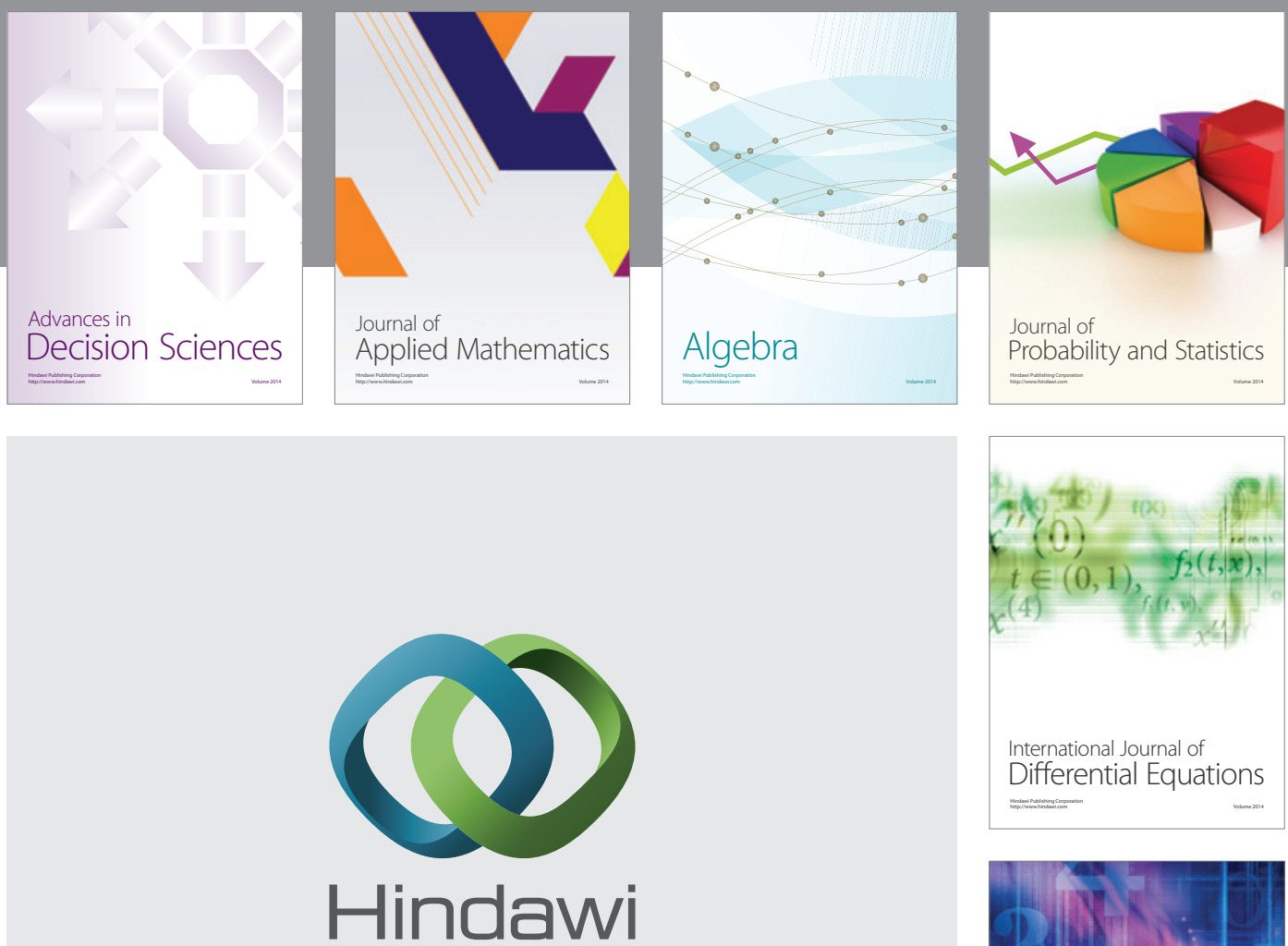

Submit your manuscripts at http://www.hindawi.com
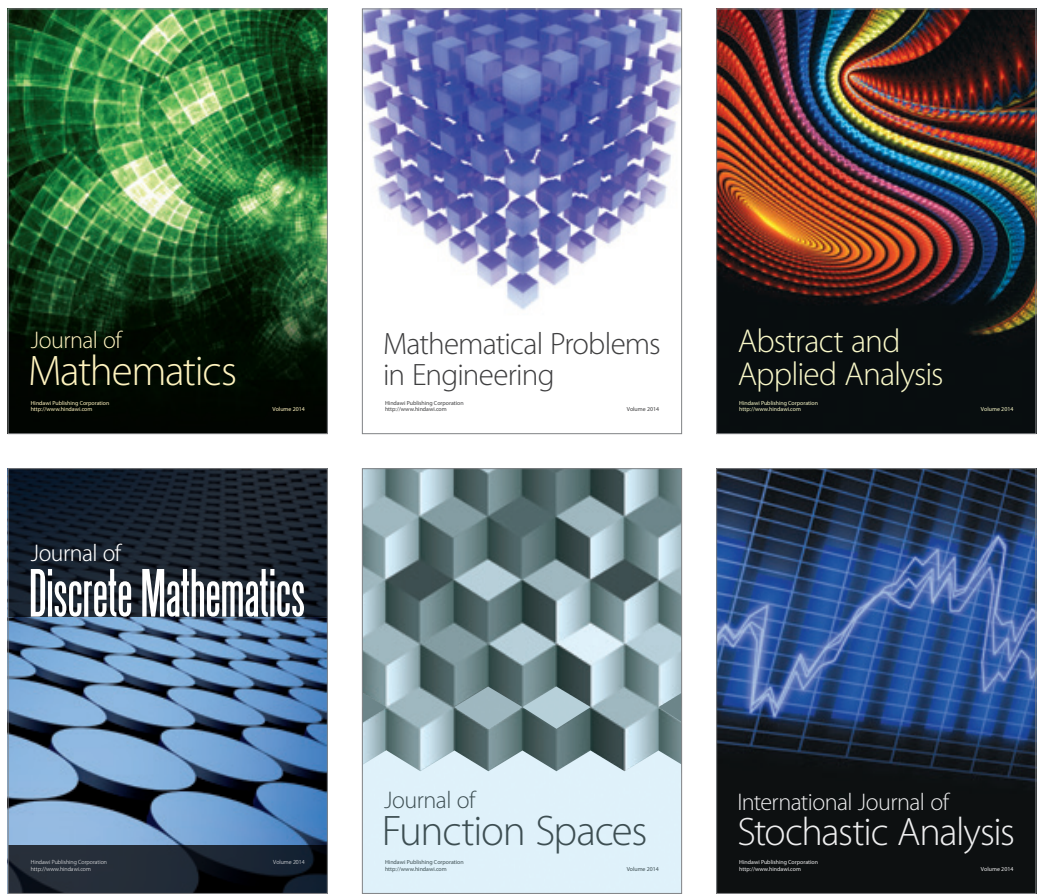

Journal of

Function Spaces

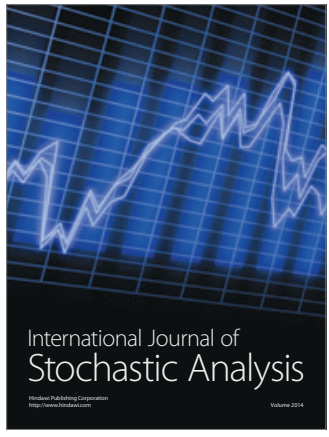

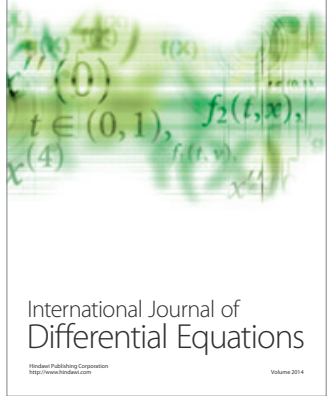
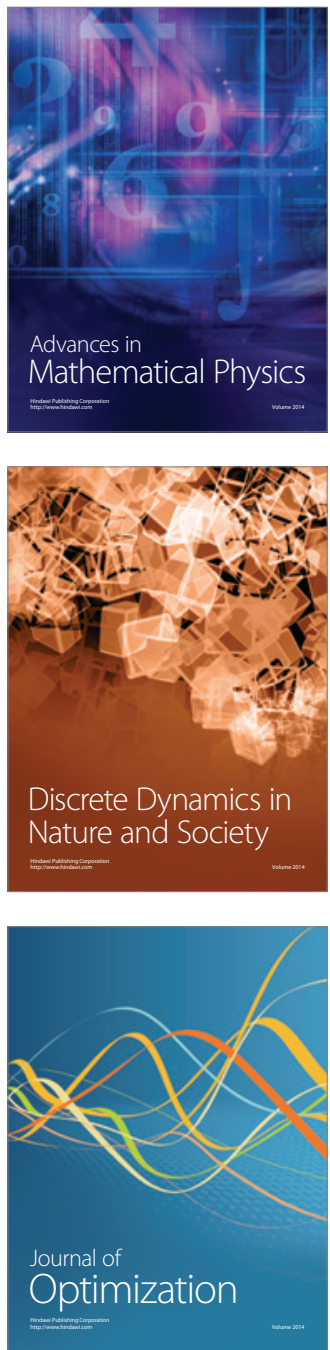